\title{
Automatic Analysis of Facial Actions: A Survey
}

\author{
Brais Martinez, Member, IEEE, Michel F. Valstar, Senior Member, IEEE, \\ Bihan Jiang, and Maja Pantic, Fellow, IEEE
}

\begin{abstract}
As one of the most comprehensive and objective ways to describe facial expressions, the Facial Action Coding System (FACS) has recently received significant attention. Over the past 30 years, extensive research has been conducted by psychologists and neuroscientists on various aspects of facial expression analysis using FACS. Automating FACS coding would make this research faster and more widely applicable, opening up new avenues to understanding how we communicate through facial expressions. Such an automated process can also potentially increase the reliability, precision and temporal resolution of coding. This paper provides a comprehensive survey of research into machine analysis of facial actions. We systematically review all components of such systems: pre-processing, feature extraction and machine coding of facial actions. In addition, the existing FACS-coded facial expression databases are summarised. Finally, challenges that have to be addressed to make automatic facial action analysis applicable in reallife situations are extensively discussed. There are two underlying motivations for us to write this survey paper: the first is to provide an up-to-date review of the existing literature, and the second is to offer some insights into the future of machine recognition of facial actions: what are the challenges and opportunities that researchers in the field face.
\end{abstract}

Index Terms-Action Unit analysis, facial expression recognition, survey

\section{INTRODUCTION}

S CIENTIFIC work on facial expressions can be traced back to at least 1862 with the work by the French researcher Duchenne [54], who studied the electro-stimulation of individual facial muscles responsible for the production of facial expressions, followed closely by the work by Charles Darwin who in 1872 published his second-most popular work 'The Expression of the Emotions in Man and Animals' [48]. He explored the importance of facial expressions for communication and described variations in facial expressions of emotions. Today, it is widely acknowledged that facial expressions serve as a primary nonverbal means for human beings to regulate their interactions [59]. They communicate emotions, clarify and emphasise what is being said, and signal comprehension, disagreement and intentions [130].

Two main approaches for facial expression measurement can be distinguished: message and sign judgement [36]. Message judgement aims to directly decode the meaning conveyed by a facial display (such as being happy, angry or

- B. Martinez and M.F. Valstar are with the Computer Vision Lab, School of Computer Science, University of Nottingham, Nottingham NG7 2RD, United Kingdom.

E-mail: \{brais.martinez, michel.valstar\}@nottingham.ac.uk.

- B. Jiang is with the IBUG group, Department of Computing, Imperial College London, London SW7 2AZ, United Kingdom. E-mail:bi.jiang09@imperial.ac.uk.

- M. Pantic is with the IBUG group, Department of Computing, Imperial College London, London, SW7 2AZ, United Kingdom and also with the Faculty of Electrical Engineering, Mathematics and Computer Science, University of Twente, Enschede 7522, NB, The Netherlands.

E-mail:m.pantic@imperial.ac.uk.

Manuscript received 5 Jan. 2016; revised 22 Mar. 2017; accepted 3 June 2017. Date of publication 0 . 0000; date of current version 0 . 0000.

(Corresponding author: Michel Valstar.)

Recommended for acceptance by J.F. Cohn.

For information on obtaining reprints of this article, please send e-mail to: reprints@ieee.org, and reference the Digital Object Identifier below.

Digital Object Identifier no. 10.1109/TAFFC.2017.2731763 sad), while sign judgement aims to study the physical sig- 35 nal used to transmit the message instead (such as raised 36 cheeks or depressed lips). Paul Ekman suggested that the 37 six basic emotions, namely anger, fear, disgust, happiness, 38 sadness and surprise, are universally transmitted through 39 prototypical facial expressions [56]. This relation underpins 40 message-judgement approaches. As a consequence, and 41 helped by the simplicity of this discrete representation, 42 prototypic facial expressions of the six basic emotions are 43 most commonly studied and represent the main message- 44 judgement approach. The major drawback of message 45 judgement approaches is that it cannot explain the full 46 range of facial expressions. Message judgement systems 47 often assume that facial expression and target behaviour 48 (e.g., emotion) have an unambiguous many-to-one corre- 49 spondence, which is not the case according to studies in 50 psychology [7] and in general, relations between messages 51 and their associated displays are not universal, with facial 52 displays and their interpretation varying from person to 53 person or even from one situation to another. 54

The most common descriptors used in sign-judgement 55 approaches are those specified by the Facial Action Coding 56 System (FACS). The FACS is a taxonomy of human facial 57 expressions. It was originally developed by [58], and 58 revised in [57]. The revision specifies 32 atomic facial mus- 59 cle actions, named Action Units (AUs), and 14 additional 60 Action Descriptors (ADs) that account for head pose, gaze 61 direction, and miscellaneous actions such as jaw thrust, 62 blow and bite. In this survey, we will limit our discussion to 63 AUs, because it is they that describe the muscle-based 64 atomic facial actions.

The FACS is comprehensive and objective, as opposed to 66 message-judgement approaches. Since any facial expression 67 results from the activation of a set of facial muscles, every 68 possible facial expression can be comprehensively described 69 


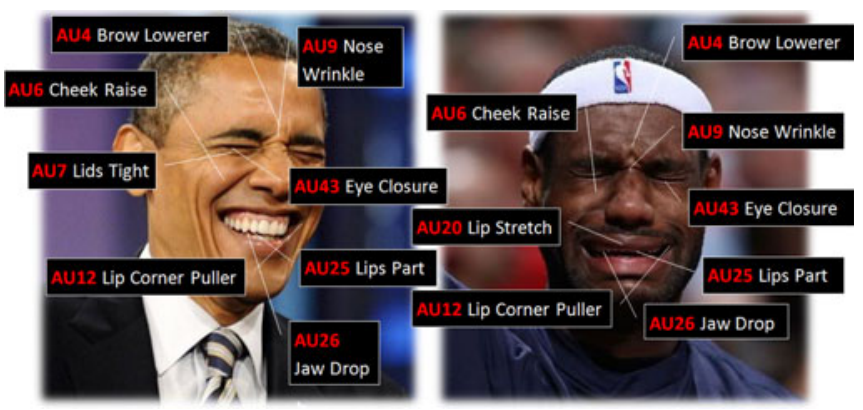

Fig. 1. Examples of upper and lower face AUs defined in the FACS.

70 as a combination of AUs [58] (as shown in Fig. 1). And while 71 it is objective in that it describes the physical appearance of 72 any facial display, it can still be used in turn to infer the sub73 jective emotional state of the subject, which cannot be 74 directly observed and depends instead on personality traits, 75 context and subjective interpretation.

Over the past 30 years, extensive research has been conducted by psychologists and neuroscientists using FACS for various aspects of facial expression analysis. For example, it has been used to demonstrate differences between polite and amused smiles [5], deception detection [63], facial signals of suicidal and non-suicidal depressed patients [76], and voluntary or evoked expressions of pain [59], [214].

Given the significant role of faces in our emotional and social lives, automating the analysis of facial signals would be very beneficial [131]. This is especially true for the analysis of AUs. A major impediment to the widespread use of FACS is the time required both to train human experts and to manually score videos. It takes over 100 hours of training to achieve minimal competency as a FACS coder, and each minute of video takes approximately one hour to score [53], [58]. It has also been argued that automatic FACS coding can potentially improve the reliability, precision, reproducibility and temporal resolution of facial measurements [53].

In spite of these facts, message-judgement approaches have been the most popular automatic approaches. This is unsurprising, however, given the complexity of the AU detection problem - a high number of classes (32 AUs versus six basic emotions), more subtle patterns, and small betweenclass differences. It is also less laborious to collect a data-set of prototypic expressions of the six basic emotions. In fact, automatic message judgement in terms of basic emotions is considered a solved problem nowadays, while machine analysis of AUs is still an open challenge [184], [186], [189].

Historically, the first attempts to automatically encode AUs in images of faces were reported by [17], [100] and [134]. The focus was on automatic recognition of AUs in static images picturing frontal-view faces, displaying facial expres- 107 sions that were posed on instruction. However, posed and 108 spontaneous expressions differ significantly in terms of their 109 facial configuration and temporal dynamics [6], [130]. 110 Recently the focus of the work in the field has shifted to auto- 111 matic AU detection in image sequences displaying spontane- 112 ous expressions (e.g., [130], [189], [214]). As a result, new 113 challenges such as head movement (including both in-plane 114 and out-of-plane rotations), speech and subtle expressions 115 have to be considered. The analysis of other aspects of facial 116 expressions such as facial intensities and dynamics has also 117 attracted increasing attention (e.g., [100], [177], [191]). 118 Another trend in facial action detection is the use of 3D infor- 119 mation (e.g., [156]). However, we limit the scope of this sur- 120 vey to 2D, and refer the reader to [151], [185] for an overview 121 of automatic facial expression analysis in 3D.

Existing works surveying methods on automatic facial 123 expression recognition either focus on message-judgement 124 approaches [61], [133], or contain just a limited subset of 125 works on automatic AU detection [174], [214], or focus on 126 the efforts of particular research groups [50], [131]. Further- 127 more, during the last 5-7 years, the field of automatic AU 128 detection produced a dramatic number of publications, and 129 the focus has turned to spontaneous expressions captured 130 in naturalistic settings. More recent surveys include [154] 131 and [43]. However, Sariyanidi et al. [154] focus mostly on 132 face representation methodologies, and touch only lightly 133 on the inference problems and methodologies. Furthermore, 134 their work is not AU-specific; since it discusses different 135 affect models. Similarly, [43] includes different data modali- 136 ties, different affect models and historical considerations on 137 the topic. Other works providing an overview of the field 138 include [35], [110], and [194], which focus primarily on 139 applications and problems related to facial AUs. This work 140 provides a comprehensive survey of recent efforts in the 141 field and focuses exclusively on automatic AU analysis 142 from RGB imagery.

We structure our survey into works on three different steps 144 involved in automatic AU analysis: 1) image pre-processing 145 including face and facial point detection and tracking, 2) facial 146 feature extraction, and 3) automatic facial action coding based 147 on the extracted features (see Fig. 2).

The remainder of the paper is structured as follows. 149 Section 2 presents a brief review of relevant issues regarding 150 FACS coding as introduced by [57]. Section 3 provides a 151 summary of research on face image pre-processing. Section 4152 contains a detailed review of recent work on facial feature 153 extraction. Section 5 summarises the state of the art in 154 machine analysis of facial actions. An overview of the 155 FACS-annotated facial expression databases is provided in 156

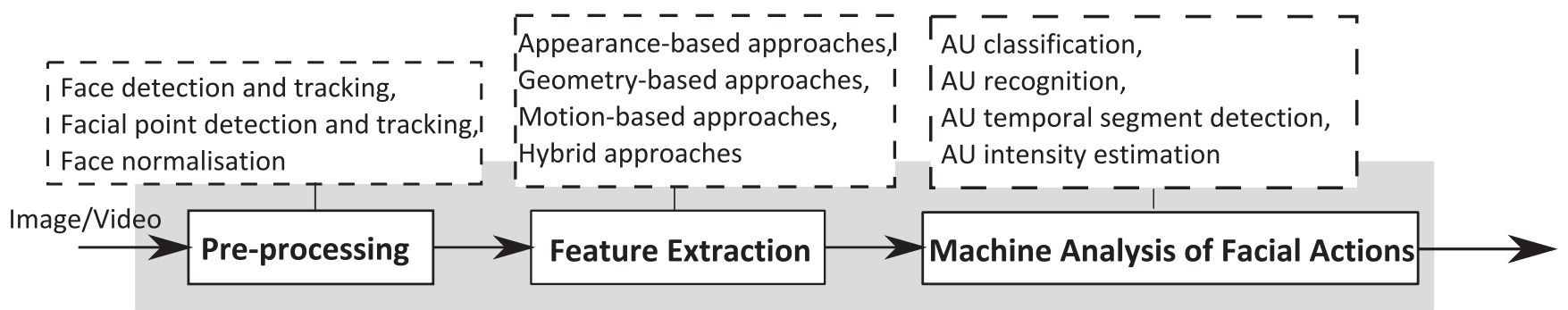

Fig. 2. Configuration of a generic facial action recognition system. 


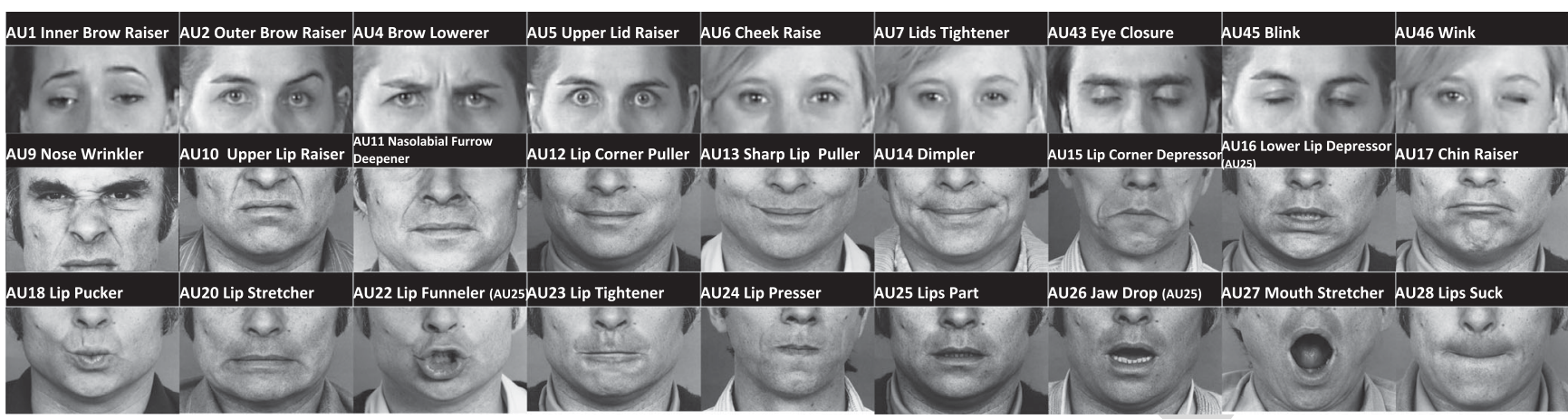

Fig. 3. A list of upper and lower face AUs and their interpretation.

Section 6. Finally, Section 7 discusses the challenges and opportunities in machine analysis of facial actions.

\section{2 facial Action Coding System (FACS)}

Here we summarise important FACS-related notions. Interested readers can find more in-depth explanations on the FACS manuals [57], [58], which formally define them.

The Facial Action Coding System [57], [58] defines 32 atomic facial muscle actions named Action Units (AUs) (as shown in Fig. 3). Additionally it encodes a number of miscellaneous actions, such as eye gaze direction and head pose, and 14 Action Descriptors for miscellaneous actions. With FACS, every possible facial expression can be objectively described as a combination of AUs. Table 1 shows a number of expressions with their associated AUs.

Voluntary versus Involuntary: The importance of distinguishing between involuntary and deliberately displayed (often referred to as "posed") facial expressions is justified by both the different semantic content of the facial expression, and the different physical realisation of the expressions ([59], [119], [142]). While one will be able to find the same AU occurrences in both voluntary and involuntary expressions, they will differ in terms of dynamics. In particular the duration of temporal phases of FACS (onset, apex, offset), the interaction between AUs (timing and co-occurrence), and the symmetry of individual AUs is different between the two categories of expressions.

$A U$ Intensity: AU intensity scoring is done on a five-point ordinal scale, A-B-C-D-E, where A refers to a trace of the action and $\mathrm{E}$ to maximum evidence.

TABLE 1

Lists of AUs Involved in Some Expressions

\section{AUs}

\begin{tabular}{ll}
\hline FACS: & $\begin{array}{l}\text { upper face: } 1,2,4-7,43,45,46 ; \\
\text { lower face: } 9-18,20,22-28 ; \text { other: } 21,31,38,39\end{array}$ \\
\hline anger: & $4,5,7,10,17,22-26$ \\
disgust: & $9,10,16,17,25,26$ \\
fear: & $1,2,4,5,20,25,26,27$ \\
happiness: & $6,12,25$ \\
sadness: & $1,4,6,11,15,17$ \\
surprise: & $1,2,5,26,27$ \\
\hline
\end{tabular}

pain: $\quad 4,6,7,9,10,12,20,25,26,27,43$

cluelessness: $\quad 1,2,5,15,17,22$

speech: $\quad 10,14,16,17,18,20,22-26,28$
Morphology and dynamics are two dual aspects of a facial 186 display. Face morphology refers to facial configuration, 187 which can be observed from static frames. Dynamics reflect 188 the temporal evolution of one facial display to another, and 189 can be observed in videos only. For example, dynamics 190 encode whether a smile is forming or disappearing. Facial 191 dynamics (i.e., timing, duration, speed of activation and 192 deactivation of various AUs) can be explicitly analysed by 193 detecting the boundaries of the temporal phase (namely neu- 194 tral, onset, apex, offset) of each AU activation. They have 195 been shown to carry important semantic information, useful 196 for a higher-level interpretation of the facial signals [6], [38]. 197

Dynamics are essential for the categorisation of complex 198 psychological states like various types of pain and mood 199 [55], [200]. They improve the judgement of observed facial 200 behaviour (e.g., affect) by enhancing the perception of 201 change and by facilitating the processing of facial configura- 202 tion. They represent a critical factor for interpretation of 203 social behaviours like social inhibition, embarrassment, 204 amusement and shame ([45], [59]). They are also a key 205 parameter in differentiating between posed and spontane- 206 ous facial displays ([65], [64], [38], [56]), and the interpreta- 207 tion of expressions in general [6].

AU combinations: More than 7,000 AU combinations have 209 been observed in everyday life [158]. Co-occurring AUs can 210 be additive, in which the appearance changes of each sepa- 211 rate AU are relatively independent, or non-additive, in 212 which one action masks another or a new and distinctive set 213 of appearances is created [57]. When these co-occurring 214 AUs affect different areas of the face, additive changes are 215 typical. By contrast, AUs affecting the same facial area are 216 often non-additive. Furthermore, some AU combinations 217 are more common than others due to latent variables such 218 as emotions. For example, happiness is often expressed as a 219 combination of AU12 and AU6.

\section{Pre-Processing}

Data pre-processing consists of all processing steps that are 222 required before the extraction of meaningful features can 223 commence. The most important aim of the pre-processing 224 step is to align faces into a common reference frame, so that 225 the features extracted from each face correspond to the 226 same semantic locations. It removes rigid head motion and, 227 to some extent, antropomorphic variations between people. 228 We distinguish three components; face localisation, facial 229 landmark localisation, and face normalisation/alignment. 


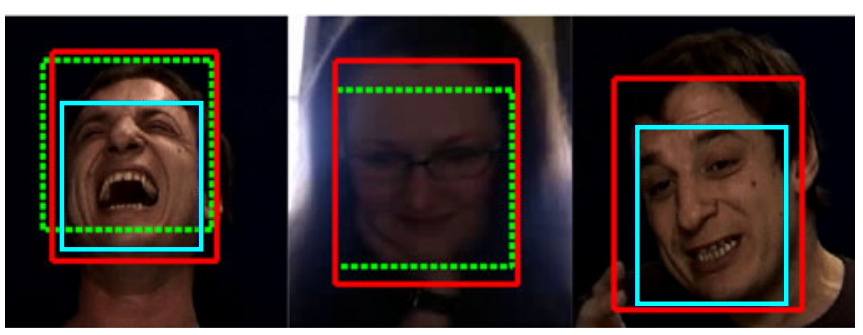

Fig. 4. Green: [196] (Matlab's implementation). Red: [128]. Blue: [227] (bounding box definition is different for each method). [196] shows less detection stability, and fail for non-frontal head poses. [227] fails to detect low quality faces.

\subsection{Face Detection}

The first step of any face analysis method is to detect the face. The Viola \& Jones (V\&J) face detector [196] is by far the most widely employed one. The public availability of pretrained models (e.g., in OpenCV or Matlab), its reliability for frontal faces and its computational simplicity makes it the reference face detection algorithm. Another popular open-source face detector is the one provided with the dlib library. ${ }^{1}$ Current automatic AU analysis methods assume a frontal head pose and a relatively controlled scenario. However, multi-view face detection algorithms will be necessary for more general scenarios.

Some recent works have successfully adapted the deformable parts model (DPM) [62] to perform face detection. This resulted in a much improved detection robustness and localisation accuracy, usually to the expense of higher computational cost. For example, [227] proposed an algorithm capable of jointly performing reliable multi-view (from -90 to 90 degree yaw rotation) face detection, head pose estimation and facial point detection. Alternatively, [128] and [112] noted that the focus on facial landmarking results in sub-optimal performance of the face detection task, proposing face-detection-specific DPM. A further speed-up was attained in [128] by adopting a cascaded detection strategy. Notably, [112] reached similar performance employing $V \& J$-like rigid-template detectors over feature channels. Source code for these works is publicly available from the respective authors' websites. Other interesting ideas have recently been proposed, as for example the use of deep learning for face detection [97]. See [211] for a recent survey on face detection. However, the current absence of publicly-available implementations detracts from their interest for those focusing on facial AU analysis. Some face detection examples are shown in Fig. 4.

\subsection{Facial Landmark Localisation}

Facial landmarks are defined as distinctive face locations, such as the corners of the eyes, centre of the bottom lip, or the tip of the nose. Taken together in sufficient numbers they define the face shape. While facial expression recognition can be attained only using the face detection, further localising the face shape results in better performance. It allows for better face registration, as well as being necessary to extract some types of features (see Section 4.2). It is common to distinguish between generative and discriminative facial landmarking algorithms, a distinction we keep here.

1. Available at: http://dlib.net/
We further discuss facial landmark tracking algorithms, 276 and include a discussion with a number of practical aspects. 277 Please note that we do not provide an exhaustive overview 278 of facial landmark detection algorithms. Instead, we discuss 279 here common trends in the research on this topic. For a 280 more exhaustive, if slightly dated, survey of facial landmark 281 detection and tracking techniques, please see [32].

\subsubsection{Generative Models}

283

Generative models are tightly identified with the active 284 appearance models (AAM) [41], [113]. The AMM finds the 285 optimal parameters for both the face shape and face appear- 286 ance that optimally reconstruct the face at hand. The land- 287 marks are provided by the reconstructed face. To this end, 288 the shape is parametrised through the widely-used Point 289 Distribution Model (PDM) [40], which relies on a PCA 290 decomposition of the shape. Then, the face shape is used to 291 define a triangular mesh, and appearance variations within 292 each triangle is again encoded using PCA. Both shape and 293 appearance can be reconstructed back-projecting their PCA 294 coefficients, and the aim is to minimise the difference 295 between the reconstructed face and the original image. 296

AAMs can be very efficient due to the use of the inverse 297 compositional for the parameter search [113]. However, 298 there has been a long-standing discussion regarding the 299 capability of AAMs to generalise to unseen faces, i.e., faces 300 of subjects not included in the training set. The performance 301 reported is often lower than for other methods in this set- 302 ting. As a consequence, several works in the AU literature 303 apply AAM in person-specific scenarios and with careful 304 landmarking initialisation, where AAM offers excellent per- 305 formance (e.g., [228]). However, recent works, such as [181], 306 [183], have shown that generic AAM can offer state-of-the- 307 art performance provided that an adequate minimisation 308 procedure is used and a good initial shape estimate is avail- 309 able. Further improvements were attained by substituting 310 the triangular mesh to represent appearance with a part- 311 based model [182], and by adopting a cascaded regression- 312 like minimisation procedure [180].

While AAM can be computationally efficient and pro- 314 vide very accurate alignments, they are not as robust as dis- 315 criminative models, and require a better initial shape 316 estimate. Furthermore, if the initial shape is outside the 317 basin of attraction of the ground truth minimum, the algo- 318 rithm might converge to a totally wrong solution.

\subsubsection{Discriminative Models}

Discriminative models typically represent the face appear- 321 ance by considering small patches around the facial land- 322 marks. For each of such patches, a feature descriptor such as 323 HOG [46] is applied, and all of the resulting descriptors are 324 concatenated into a single vector to create the face represen- 325 tation. Discriminative methods proceed by training either a 326 classifier or a regressor on these features. There is a wide 327 variety of discriminative facial landmarking algorithms. In 328 here we distinguish three sub-families, response-map fitting, 329 deformable parts model and regression-based approaches. $\quad 330$

Response map fitting: which includes the popular Active 331 Shape Model [42] and its variants, have been very popular 332 due to their early success and the availability of well- 333 
optimised public implementations of some of its most popular variants [120], [153]. These methods divide the landmarking process into two distinct steps. In the first step, model responses are computed in the vicinity of the current landmark location, encoding the belief of the appearance model of each evaluated location being the true landmark location. The second step consists of finding the valid shape that maximises the combined individual responses. These two steps are alternated iteratively until convergence.

Responses have traditionally been computed using classifiers trained to distinguish between the true landmark location and its surroundings, using either a probabilistic output (e.g., logistic regression) or some confidence measure like the SVM margin [153]. However, some recent works have shown it is possible to construct similar responses from regressors, providing better performance ([39], [111]). This can be done by training a regression model to predict the displacement from the test location to the true landmark location. Then, at test time, the regressor is evaluated on a set of test locations (e.g., a regular grid), and the resulting predictions are combined to create the responses.

The second step consists of finding the valid shape that maximises the sum of the individual responses. This is however very challenging, with frequent convergence to local minima. Thus, much of the research drive has been focused on improving the shape fitting step. For example, [20] proposed a shape fitting step that used exemplars in a RANSAC manner, while [12] proposed to use a regression strategy to directly find increments to the shape parameters that maximise the combined responses.

More recently, CNN methods have shown significant success when used to produce the response maps. The response map creation and the shape fitting can then both be combined into an end-to-end training [78].

Regression-based methods bypass the construction of the response maps by directly estimating the difference between the current shape estimate and the ground truth. This estimation is carried out by discriminative regression models, trained with large quantities of ground-truth shape perturbations. The excellent performance attained by regression-based methods relies on two factors. First, they incorporate the cascaded regression approach [52], so that the shape estimation results from the application of a fixed succession of regressors, each one tuned to the output of the previous regressor. Second, the direct estimation of the shape is targeting, bypassing the construction of response maps. Thus, the complex constrained response map maximisation step is avoided.

Initially proposed by [24], [25], much of the popularity of regression-based approaches is due to the Supervised Descent Method (SDM) [203]. This is due to the simplicity of the method, as the final estimate is computed using only four matrix multiplications, feature computation and face detection aside. Other variants of this methodology subsequently attained remarkable results. For example, [25], [93], [140] proposed extremely efficient variants relying on regression forest for inference. An extension of SDM to deal with large head pose variation, including profile views, was proposed in [204]. Yan et al. [205] proposed an algorithm capable of robustly combining multiple SDM-based fittings, of particular importance on more challenging scenarios.
Jeni et al. proposed a cascade regression approach that 395 makes predictions of 3D shapes from 2D images [85]. 396 Finally, Burgos-Artizzu et al. [23] focused on improving 397 performance under partial occlusion. Tzimiropoulos [180] 398 proposed instead to use the discriminatively-trained regres- 399 sion cascade with the generative model proposed in [182], 400 resulting in a large performance gain. The most accurate 401 facial point localisation technique at time of writing is incre- 402 mental Continuous Cascaded Regression (iCCR, [149]), 403 which replaces sampling-based regression with an analyti- 404 cal solution that integrates over all evidence in an area of 405 the image approximated by a Taylor expansion of the 406 appearance descriptors.

Deep learning methods have also been successfully 408 applied to face alignment. For example, [168] proposed a 409 cascaded regression deep-learning landmarking methodol- 410 ogy. Subsequently, [220] further leverages auxiliary face 411 analysis tasks such as smile detection and head pose estima- 412 tion to improve upon the prediction accuracy. Instead, [226] 413 proposed a methodology for dealing with larger non-frontal 414 head pose variation by probing the space shape to find a 415 good shape to regress from rather than using a pre-defined 416 mean shape as the starting point. Finally, [178] cast the cas- 417 caded regression as a Recurrent CNN and performed end- 418 to-end training of the cascade.

Deformable Parts Models, first introduced by [227] for 420 facial landmarking, are strongly related to the response- 421 map fitting methods. However, they boast a unique prop- 422 erty: they reach globally optimal fittings. This is achieved 423 by using a tree graph to perform a soft constraint on the 424 face shape, e.g., flat chain [227] or a hierarchical tree [67]. 425 Both shape and appearance are integrated into a single loss 426 function which can be minimised efficiently and exactly for 427 inference. However, the sheer number of possible outputs 428 makes detection very slow if the image is large. Further- 429 more, the soft shape constrains results in lower detection 430 precision when compared to other state-of-the-art methods. 431 Thus, these methods can be used for initialising regression- 432 based landmarking methods, provided there are no real- 433 time performance constraints [180].

\subsubsection{Facial Landmark Tracking}

When facial landmark localisation on a full sequence is 436 desired, a landmark detection algorithm can be applied on 437 each individual frame. This however neglects important 438 temporal correlations between frames. The previous detec- 439 tion can be used as the initial shape on the current frame, 440 leading to a much better estimate. Also, models can be 441 trained specifically for the tracking case, leading to 442 improved performance, as shown in for the standard SDM 443 case [203], and in [204] for the global SDM, which can 444 include up-to-profile head rotation. Furthermore, sequen- 445 tial data allows for the on-line update of the appearance 446 models. In this way, the appearance model is incremen- 447 tally adapted to the specific characteristics of the test 448 sequence. This was exploited by [11], which proposed an 449 extension of [203] capable of performing incremental 450 learning. [138] proposed an alternative adaptation strategy 451 based on subspace learning. Further advances were 452 attained by Sanchez Lozano et al. [149], who use a variant 453 of linear regression in iCCR that is used to reduce the 454 


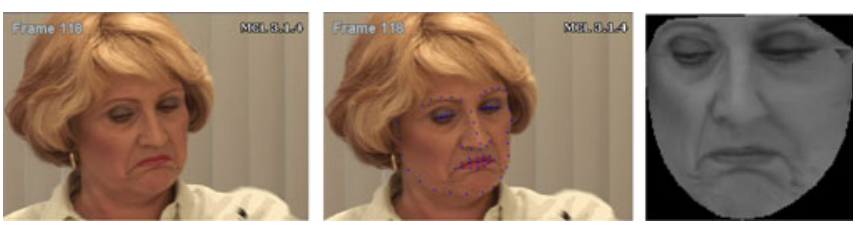

Fig. 5. Original face (left), AAM tracking result (centre), result of texture warping to the mean shape (right). The right part of the nose and face are not reconstructed properly due to self-occlusions. There is residual expression texture (right). Images taken from UNBC-McMaster shoulder pain database, tracking results by [90].

computational complexity of the incremental updates, resulting in what is to date the only real-time tracking with incremental learning.

Finally, for applications where an offline analysis is possible, techniques such as image congealing can be applied in order to remove tracking errors [147]. CNNs have also been applied to this problem, notably in [137], which relies on Recurrent NN. However, the performance improvement is limited for near-frontal head poses (typical for current AU analysis problems), so that the increased computational resources required might be an important drawback in this case. The 300 Videos in the Wild [163] is currently the bestestablished benchmark on this topic. It provides performance in three categories corresponding to different levels of complexity.

\subsection{Face Registration}

Face registration aims at registering each face to a common pre-defined reference coordinate system. The information obtained on the face alignment stages can be used to compute such a transformation, which is then applied to the image to produce the registered face. The rationale is that misalignments produce large variations in the face appearance and result in large intra-class variance, thus hindering learning. In here we provide a short overview of the possible approaches. We refer the interested reader to [154] for further details, as it already provides a complete and adequate coverage of this topic.

Procrustes. A Procrustes transformation can be used to eliminate in-plane rotation, isotropic scaling and translation. While translation and scaling can be computed using only the face bounding box, this result can be imprecise, and the use the facial landmarks can provide much better results (e.g., [176], [88], [228]).

Piecewise Affine. After detecting the facial landmarks, they are put in correspondence to some pre-defined shape (e.g., a neutral face). By defining a triangular mesh over face shapes, each triangle can be transformed according to the affine transformation define by its vertices. This yields a strong registration, although it produces the loss of some expressive information. In some cases, data corruption can be introduced (see Fig. 5). Face frontalisation is currently receiving a lot of attention [74], [124], [148], and some of the novel methods might lead to improvements.

Finally, some works report performance improvements using piecewise affine face registration when compared to a standard Procrustes registration by combining the resulting appearance with some geometric information capturing the landmark configuration prior to the registration (see Section 4) [10], [30], [31].

\subsection{Discussion}

Very recent advances on face detection can yield much better 505 performance than the Viola and Jones algorithm. For exam- 506 ple, [112] is publicly available from the authors' web pages 507 and offers excellent performance and is computationally 508 light. When it comes to facial landmarking, a tracking algo- 509 rithm is desired, as it can offer much more stable detections. 510 Regression-based methods are nowadays the most robust 511 ones. While other methods can achieve better performance 512 in more complex scenarios, [203] offers an excellent trade-off 513 of implementation simplicity and effective inference for up 514 to 30 degree of head rotation. The authors of [11] also offer a 515 publicly available implementation of their incremental track- 516 ing algorithm. If extremely low computational cost is 517 desired, then [140] can yield reliable detection at up to 3,000 518 fps, although its implementation is far from straightforward. 519

Implementing a Procrustes registration is straightfor- 520 ward. More complex models aiming to remove non-frontal 521 head poses are more complex and artefact prone. It is how- 522 ever an interesting component for ongoing research. 523

Constructing an integrated and robust system that per- 524 forms real facial landmark tracking in (near) real time was 525 the most recently solved problem. Notably, iCCR has pre- 526 sented a faster than real-time tracker with incremental 527 learning, code for which is available for research [149]. 528 OpenFace [14] also constitutes an effort along these lines. It 529 is an open source real-time software implementing the full 530 pipeline for facial AU recognition from video, including 531 face alignment and head pose estimation.

Temporally smoothing the predictions, and model adap- 533 tation are other interesting aspects that require more atten- 534 tion. A working system under occlusions is also an open 535 problem. While some landmarking methods are robust to 536 occlusions, further work is required in this direction. The 537 ideal method would not only be accurate under occlusions, 538 but also explicitly detect them, so that this information can 539 be taken into account by subsequent processing layers.

\section{Feature Extraction}

Feature extraction converts image pixel data into a higher- 542 level representation of motion, appearance and/or the spa- 543 tial arrangement of inner facial structures. It aims to reduce 544 the dimensionality of the input space, to minimise the vari- 545 ance in the data caused by unwanted conditions such as 546 lighting, alignment errors or (motion) blur, and to reduce 547 the sensitivity to contextual effects such as identity and 548 head pose. Here, we group the feature extraction methods 549 into four categories: appearance-based, geometry-based, 550 motion-based and hybrid methods. Another thorough sur- 551 vey of face features was presented by Sariyanidi et al. [154]. 552

\subsection{Appearance Features}

Appearance features describe the colour and texture of a 554 facial region and are nowadays the most commonly used fea- 555 tures. They can be used to analyse any given AU, and they 556 encompass a wide range of designs of varying properties. 557 This offers researchers flexibility and room for methodologi- 558 cal improvements. However, appearance features can be 559 sensitive to non-frontal head poses and to illumination 560 changes. Appearance features can be characterised in terms 561 


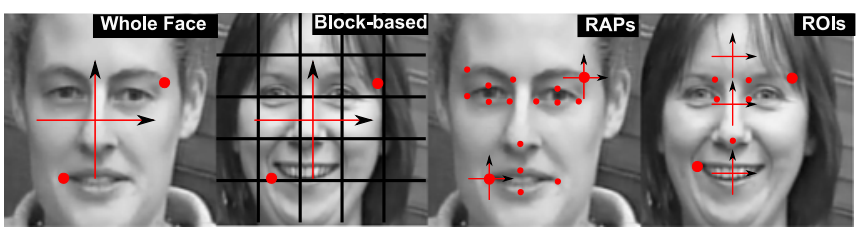

Fig. 6. Different ways to apply appearance descriptors. Left to right: whole face, block-based, Region Around Points (RAPs) and Region Of Interests (ROIs) defined by points. The first two representations are holistic, while the second two are local.

of the representation strategy (what part of the face they represent), the feature type (which features are used to represent it), and whether the features are static (encode one single frame) or dynamic (encode a spatio-temporal volume).

Representation strategy: Appearance features can be extracted from the whole face (holistic features) or from specific face regions defined by inner facial structures (local features). More precisely, we define holistic features as those that extract information according to a coordinate system relative to the entire face (e.g., [215]). In contrast, local methods consider locations relative to a coordinate system defined by inner-facial features such as facial components or facial points (e.g., [177]).

The most typical local approach considers small patches centred around each of the facial landmarks or a subset of them. Then, for each of the patches, a feature descriptor is applied, and the resulting descriptors are concatenated into the final feature vector. Instead, holistic approaches represent the whole face region, for example as given by the bounding box. However, many approaches use a blockbased representation, by which the face region is divided into a regular grid of non-overlapping blocks, and features are then extracted from each block and concatenated into a single vector (e.g., [88]). This process is sometimes also referred to as tiling. Many feature descriptors use histograms taken over the contents of the blocks to increase shift robustness, as histograms eliminate the spatial arrangements. However, histogramming over the whole face region would eliminate too much information regarding spatial arrangements of the features, thus the resorting to tiling. It is interesting to note that according to our definition, blockbased methods are still holistic, as they do not use inner facial structures to define what to represent. Fig. 6 shows an illustration of the different approaches.

The desired properties of the features vary when using holistic or local approaches. For holistic methods, the level of correspondence between two faces is relatively poor, i.e., each feature dimension will typically relate to a different point in the face. Instead, local methods show better registration properties. Thus, robustness to misalignment is more important for the former. Local representations have other important advantages; illumination changes can locally be approximated as homogeneous, which enables them to be normalised easily, and non-frontal head poses can be locally approximated by an affine transformation. Instead, holistic approaches have the more complex task of dealing with the global effect of these changes. With face registration now being very accurate, local representations are generally to be preferred.

Appearance feature types in the automatic AU analysis literature can be divided into five categories: intensity, filter banks, binarised local texture, gradient-based, and two- 613 layer descriptors. Each comprises several different related 614 feature types, and shares important properties.

Image intensity: Some works have advocated for the use of 616 raw pixel intensities as the preferred appearance feature 617 (e.g., [31], [106], [108]). They proposed to overcome the sen- 618 sitivity to head-pose variation by performing precise facial 619 landmarking, and then applying a piecewise affine transfor- 620 mation, obtaining a strong registration (e.g., by [30]) (see 621 Section 3.3). An extension was proposed in [121], where a 622 feature representation based on pixel intensities was learnt. 623 To this end, the authors used a discriminative sparse dictio- 624 nary learning technique based on a piecewise affine strong 625 registration for intensity estimation. However, pixel intensi- 626 ties are sensitive to all kinds of distractor variation. While 627 reported experiments show that image intensity offers com- 628 petitive performance, the evaluation datasets used do not 629 contain illumination variations and these results might not 630 generalise (something forewarned by [30]). Non-frontal 631 head poses are in this case problematic as the registration 632 often produces artefacts. Since the piecewise affine registra- 633 tions eliminates important shape information, the authors 634 advise combining intensity and geometric features (see 635 below) to compensate the information loss. 636

Filter banks: These features result from convolving every 637 location of a region with a set of filters. While they have 638 strong expressive power, they lack some robustness to 639 affine transformations and illumination changes.

Gabor wavelets are common in the field of automatic AU 641 analysis (especially in early works), as they are sensitive to 642 fine wave-like image structures such as those corresponding 643 to wrinkles and bulges. Only Gabor magnitudes are typi- 644 cally used (i.e., Gabor orientation is discarded), as they are 645 robust to small registration errors. Being sensitive to finer 646 image structures, they can be a powerful representation, 647 provided that the parametrisation is correct, i.e., filters have 648 to be small enough to capture more subtle structures. How- 649 ever, the resulting dimensionality is very large, especially 650 for holistic approaches and the high computational cost is a 651 burden for real-time applications. ${ }^{2}$ A typical parametrisa- 652 tion consists of 8 orientations, and a number of frequencies 653 ranging from 5 to 9 . Due to their representational power, 654 Gabor filters have recently been used as a component of 655 two-layer feature representations (see below).

Other filters within this category include the Discrete 657 Cosine Transform (DCT) features [1] and Haar-like features 658 [136]. DCT features encode texture frequency using prede- 659 fined filters that depend on the patch size. DCTs are not sen- 660 sitive to alignment errors, and their dimensionality is the 661 same as the original image. However, higher frequency 662 coefficients are usually ignored, therefore potentially losing 663 sensitivity to finer image structures such as wrinkles and 664 bulges. Furthermore, they are not robust to affine transfor- 665 mations. Haar-like filters, employed in [199] for facial AU 666 detection, fail to capture finer appearance structures, and 667 their only advantage is their computational efficiency. Thus, 668 their use should be avoided, or limited to detecting the 669 most obvious AUs (e.g., AU12).

2. If only inner products of Gabor responses are needed, then very significant speed ups can be attained [9]. 


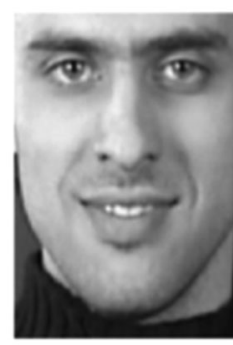

(a)

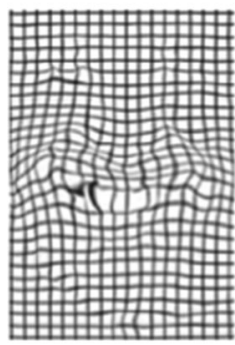

(d)

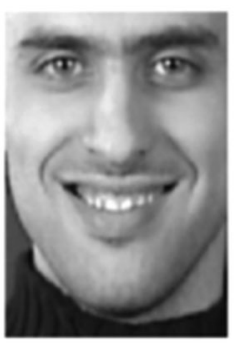

(b)

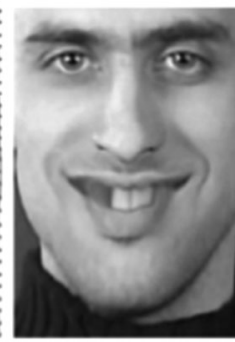

(e)

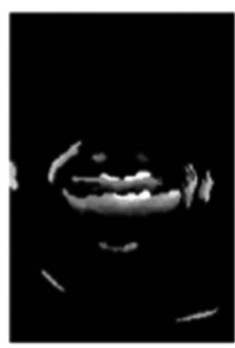

(c)

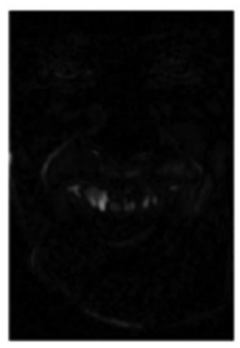

(f)
Fig. 7. Example of MHI and FFD techniques. (a) First frame. (b) Last frame. (c) $\mathrm{MHI}$ for the entire sequence. (d) The motion field sequence from the FFD method applied to a rectangular grid. (e) The motion field sequence from the FFD method applied to the first frame. (f) Difference between (b) and (e). [94]. Image taken from Koelstra et al. [94].
Binarised local texture: Local Binary Patterns (LBP) [125] and Local Phase Quantisation (LPQ) [127] are popular for automatic AU analysis. Their properties result from two design characteristics: 1) real-valued measurements extracted from the image intensities are quantised to increase robustness, especially to illumination conditions, 2) histograms are used to increase the robustness to misalignment, at the cost of some spatial information loss. Their strong robustness to illumination changes and misalignment makes them very suitable for holistic representations, and they are typically used in a block-based manner.

The standard LBP descriptor [125] is constructed by considering, for each pixel, an 8-dimensional binary vector. Each binary value encodes whether the intensity of the central pixel is larger than each of the neighbouring pixels. A histogram is then computed, where each bin corresponds to one of the different possible binary patterns, resulting in a 256-dimensional descriptor. However, the so called uniform LBP is often used. It results from eliminating a number of pre-defined bins from the LBP histogram that do not encode strong edges [126].

Many works successfully use LBP features for automatic facial AU analysis in a block-based holistic manner (e.g., [29], [88], [202]), and the latter found $10 \times 10$ blocks to be optimal in their case for uniform LBPs. The main advantages of LBP features are their robustness to illumination changes, their computational simplicity, and their sensitivity to local structures while remaining robust to shifts [162]. They are, however, not robust to rotations, and a correct normalisation of the face to an upright position is necessary. Many variants of the original LBP descriptor exist, and a review of LBP-based descriptors can be found in [79].

The LPQ descriptor [127] uses local phase information extracted using 2D short-term Fourier transform (STFT) computed over a rectangular $\mathrm{M}$-by-M neighbourhood at each pixel position. It is robust to image blurring produced by a point spread function. The phase information in the 707 Fourier coefficient is quantised by keeping the signs of the 708 real and imaginary parts of each component. LPQs were 709 used for automatic AU analysis in [88], which found that 710 when applied in a block-based holistic manner, $4 \times 4$ blocks 711 performs the best.

Gradient-based descriptors, such as HOG [46], SIFT [104] or 713 DAISY [175], use a histogram to encode the gradient infor- 714 mation of the represented patch. Each image patch is 715 divided into blocks, and a histogram represents the orienta- 716 tion and magnitude of gradients within each block. The 717 resulting histogram is normalised to 1 , thus eliminating the 718 effect of uniform illumination variations. These features are 719 robust to misalignment, uniform illumination variations, 720 and affine transformations. However, larger gradients cor- 721 responding to facial component structures can be grouped 722 together with smaller gradients such as those produced by 723 wrinkles and bulges. Therefore, these features should be 724 applied locally to avoid larger gradients dominating the 725 representation. They offer very good robustness properties 726 when used as local features, make them one of the best (and 727 preferred) choices in the literature [33], [164], [224], [228]). 728 As an exception, [31] used HOG features in a holistic man- 729 ner, showing comparable performance to Gabor filters and 730 raw pixel information. However, the face was normalised to 731 $48 \times 48$ pixels in this study, meaning smaller structures 732 could not be captured by the alternative representations. $\quad 733$

Two-layer appearance descriptors result from the applica- 734 tion of two traditional feature descriptors, where the second 735 descriptor is applied over the response of the first one. For 736 example, [161] and [4] used Local Gabor Binary Pattern 737 (LGBP) [216]. They result from first calculating Gabor mag- 738 nitudes over the image and then applying an LBP operator 739 over the multiple resulting Gabor response maps. Gabor 740 features are applied first to capture local structures, while 741 the LBP operator increases the robustness to misalignment 742 and illumination changes and reduces the feature 743 dimensionality. In fact, [161] won the FERA2011 AU detec- 744 tion challenge with a combination of LGBP and geometric 745 features [189], making a strong case for their use. Alterna- 746 tively, [202] used two layers of Gabor features $\left(G^{2}\right)$ to 747 encode image textures that go beyond edges and bars. They 748 also compared single layer (LBP, Gabor) and dual layer $\left(G^{2}, 749\right.$ LGBP) architectures for automatic AU detection, and con- 750 cluded that two-layer architectures provide a small but con- 751 sistent improvement.

Spatio-temporal appearance features encode the appearance 753 information of a set of consecutive frames rather than only 754 that of a single frame. Such features can be used to represent 755 a single frame, typically the frame in the middle of the spa- 756 tio-temporal window [88]. This results in an enhanced 757 representation of the frame including its temporal context. 758 This strategy has been shown to work well in practice, and 759 its use is particularly justifiable since the inference target is 760 an action. Note that this category is distinct from motion fea- 761 tures, which are described in Section 4.3.

Different spatio-temporal extensions of frame-based fea- 763 tures have been devised. Notably, LBPs were extended to 764 represent spatio-temporal volumes by [222]. To make the 765 approach computationally simple, a spatio-temporal vol- 766 ume is described by computing LBP features only on Three 767 
Orthogonal Planes (TOP): XY, XT, and YT. The so-called LBP-TOP descriptor results from concatenating these three feature vectors. The same strategy was subsequently followed to extend other features, such as LPQ [88] and LGBP features [4]. The resulting representations tend to be more effective, as shown by the significant performance improvement consistently reported [4], [88], [222]. A notable property of TOP features is that the spatio-temporal features are computed over fixed-length temporal windows, so that different speeds of AUs produce different patterns.

An alternative strategy was used to extend Haar-like features to represent spatio-temporal volumes in [208]. In this case, a normal distribution models the values of each Haarlike feature per AU. Then the Mahalanobis distance for each feature value in a temporal window is computed and thresholded to create a binary pattern. The authors showed a significant performance increase when using dynamic descriptors compared to the static Haar features. However, the AU dataset used to report their results is not publicly available and is of unknown characteristics.

It is possible to abandon the frame-based representation and use spatio-temporal descriptors to analyse full facial actions, in a strategy often called segment-level analysis. This implies representing the event as a fixed length feature vector, which constrains the representation. For example, [164] and [51] use a histogram of temporal words [123], a temporal analogy to the classical bag-of-words representation [165]. In particular, [51] successfully combines featurelevel and segment-level classifiers, arguing that both models are likely to behave in a complementary manner. Segmentlevel features have the potential to capture more global patterns. However, it is not clear how to effectively represent a video segment of varying length, despite some recent efforts regarding temporal alignment [83], [87].

\subsection{Geometric Features}

Geometric features capture statistics derived from the location of facial landmarks, with most facial muscle activations resulting in their displacement. For example, facial actions can raise/lower the corner of the eyebrows or elongate/ shorten the mouth. Reliably obtaining facial point locations has traditionally been a major problem when using geometric features. However, recent breakthroughs on facial landmarking mean that geometric features in realistic scenarios can now be computed reliably.

Geometric features are easy to register, independent of lighting conditions, and yield particularly good performance for some AUs. However, they are unable to capture AUs that do not cause landmark displacements. Thus, combining geometric features with appearance features normally results in improved performance (see Section 4.5).

\subsection{Motion Features}

Motion features capture flexible deformations of the skin caused by the contraction of facial muscles. As opposed to geometric features, they are related to dense motion rather than to the motion of a discrete set of facial landmarks. They are also different from (dynamic) appearance features as they do not capture appearance but only appearance changes, so they would not respond to an active AU if it is not undergoing any change (e.g., at the apex of an expres- 826 sion). Motion features are less person specific than appear- 827 ance features. However, they require the full elimination of 828 rigid motion. This means that they are affected by misalign- 829 ment and varying illumination conditions.

We distinguish two classes of motion-based features: 831 those resulting from image subtraction, and those where a 832 dense registration at the pixel level is required.

Image subtraction: $\delta$-images are defined as the difference 834 between the current frame and an expressionless-face frame 835 of the same subject. In the early AU literature, $\delta$-images 836 were commonly combined with linear manifold learning to 837 eliminate the effect of noise; for example [16], [53], [60], and 838 [19] combined $\delta$-images with techniques such as PCA or 839 ICA. Alternatively, [53] and [19] used Gabor features 840 extracted over $\delta$-images. More recently, [95] and [156] com- 841 bined $\delta$-images with variants of Non-negative Matrix Fac- 842 torization (NMF). Finally, [195] used head-pose-normalised 843 face images to construct the $\delta$-images. Again, the use of 844 $\delta$-images relies on the first frame of the sequence being 845 neutral, which was a common bias in early databases. Some 846 very recent works have given a spin to this idea and intro- 847 duce a module predicting the neutral face at test time [13], 848 [72]. This approach [13] won the FERA 2015 pre-segmented 849 AU intensity estimation sub-challenge.

Motion History/Energy Images (MHI/MEI) [22] use 851 image differences to summarise the motion over a number of 852 frames. MEIs are binary images that indicate whether any 853 pixel differences have occurred over a given fixed number of 854 frames. In MHI, recent motion is represented by high inten- 855 sity values, while the pixels where motion was detected lon- 856 ger ago fade to zero intensity linearly over time. This was 857 first applied to AU analysis in [192], where MHI summarised 858 window-based chunks of video. An extension of MHI-based 859 representation was applied for automatic AU analysis in 860 [94], where the authors approximate the motion field by find- 861 ing the closest non-static pixel. The authors claim that this 862 results in a more dense and informative representation of the 863 occurrence and the direction of motion. The main advantage 864 of MHI-based methods is that they are robust to the inter- 865 sequence variations in illumination and skin colour. How- 866 ever they cannot extract motion directions, and are very sen- 867 sitive to errors in face registration.

Non-rigid registration: Methods based on non-rigid image 869 registration consider the direction and intensity of the 870 motion for every pixel. Motion estimates obtained by optical 871 flow $(\mathrm{OF})$ were considered as an alternative to $\delta$-images in 872 early works ([53], [101]). Koelstra et al. substituted the OF by 873 a free form deformation (FFD, [94]), and used a quadtree 874 decomposition to concentrate on the most relevant parts of 875 the face region, resulting in a large performance increase. 876 However, non-rigid registration approaches rely on the qual- 877 ity of the registration, they are complex to implement, and 878 have very high computational cost. Their use in practical 879 applications is thus not straightforward.

\subsection{Deeply Learnt Features}

While most CV problems have seen revolutionary perfor- 882 mance increases from adopting deep learning, automatic 883 AU analysis has only seen moderate benefits. Potential 884 explanations include the lack of large quantities of training 885 
data, and that there is no standard face-specific ImageNetlike pre-trained model to start fine-tuning from. The fact that deep learning has been successful for prototypical facial expression recognition [89] is promising. However, this success relied on the authors annotating very large amounts of data. An alternative to dealing with a low quantity of labelled examples is the use of transfer learning techniques [122]. While dealing with prototypical expressions, these works underpin both the potential of deep learning methods for AU analysis and the associated challenges.

Yet, some recent works have leveraged deep learning for AU analysis with increasing success. For example, [71] attained reasonable performance on the FERA 2015 challenge using standard deeply learnt features, and Jaiswal et al. who presented a novel deep learning-based representation encoding dynamic appearance and face shape [81] attained state-of-the-art results on that database.

\subsection{Combining Different Features}

Several works investigate whether geometric or appearance features are more informative for automatic AU analysis [193], [221]. However, both types convey complementary information and would therefore be best used together, and experimental evidence consistently shows that combining geometric and appearance features is beneficial [73], [95], [228]. In particular, [160] won the FERA 2011 AU detection challenge with this approach. Combining these features is even more important when using a piecewise-affine image registration (see Section 3.3), which eliminates the shape information from registered face image. Geometric features can then add back some of the information eliminated by the registration [106], [108].

Different approaches can be used to combine features of a diverse nature. Feature-level fusion is the most common [70], [73], [108], [195], [224]. It consists of concatenating different feature vectors containing different feature types into a single vector, which is then directly used as input to the learning algorithm. Decision-level fusion (e.g., [106]) proceeds instead by applying a learning algorithm to each type of features independently, and then combining the different outputs into a final prediction. For example, [106] trained two linear SVMs, over appearance and geometric features respectively, and then used the SVM margins and linear logistic regression to fuse the two outputs.

Instead, [161] recently applied the Multi-Kernel SVM framework for automatic AU analysis, and combined LGBP features with AAM shape coefficients. In this framework a set of non-linear classification boundaries are computed for each of the feature types, and the resulting scores are combined linearly in a manner typical of decision-level fusion. However, the parameters of the classifiers and the linear combination of the individual outputs are jointly minimised. In the absence of overfitting, the resulting performance will be equal or higher to that of a single feature type for every AU. This is a great advantage over feature-level fusion or decision-level fusion, where an under-performing feature type will most likely penalise the combined performance.

\subsection{Discussion}

Fuse heterogeneous features: It is in general advised to use both appearance and geometric features. Simple strategies
TABLE 2

Division of Methods According to Their Output

\begin{tabular}{lll}
\hline Problem & \multicolumn{1}{c}{ Variants } & \multicolumn{1}{c}{ Output space } \\
\hline Class. & No AU Co-ocur. & $\mathcal{Y}=\{1: k\}$ per seq. \\
\hline AU Co-ocurence & $\mathcal{Y}=\{ \pm 1\}^{k}$ per seq. \\
\hline Detection & $\begin{array}{l}\text { Frame-based inf. } \\
\text { Segment-based inf. }\end{array}$ & $\mathcal{Y}=\{ \pm 1\}^{k}$ per fr. \\
\hline Intensity & $\begin{array}{l}\text { Multiclass } \\
\text { Ordinal reg. }\end{array}$ & $\mathcal{Y}=\{0: 5\}^{k}$ per fr. \\
& Regression & $\mathcal{Y}=[0,5]^{k}$ per fr. \\
\hline Temp. seg. & Class. & $\mathcal{Y}=\{0: 3\}^{k}$ per fr. \\
\hline
\end{tabular}

$k$ indicates the number of AUs considered.

like feature-level fusion or even decision-level fusion per- 945 form well in practice. The Multiple Kernel Learning frame- 946 work is particularly well-suited for their combination. 947

Best appearance features: LBP or LPQ as a holistic represen- 948 tation, or HOG as a local representation are both good 949 choices. Gabor can be used in either of the representations, 950 but they are more computationally expensive. LGBP fea- 951 tures can be very effective too. Spatio-temporal appearance 952 features provide a consistent and significant advantage, and 953 they can be relatively efficient too.

Best geometric features: Little evidence has been presented 955 about this. Geometric features do not offer much room for 956 new feature types. Thus, optimising the set of geometric fea- 957 tures has received very little attention in the literature. After 958 face tracking, geometric features are inexpensive to com- 959 pute, so they can be attractive for problems requiring low 960 computational cost solutions.

Opportunities and directions: Further use of Deep Learn- 962 ing, in particular CNNs, is an obvious current research 963 focus. Some of the new directions on feature design point to 964 the inclusion of spatio-temporal context (and other sources 965 of context) in the feature construction. How to best combine 966 different features, including mixtures of learned and hand- 967 crafted features is an open question. Finally, what features 968 are best for low-intensity expressions is another interesting 969 open question.

\section{Machine Analysis of Facial Actions}

In this section we review different machine learning techni- 972 ques applied to various AU-related problems. We distin- 973 guish four problems: AU detection, AU intensity 974 estimation, AU temporal segment detection and AU classifi- 975 cation (see Table 2). The aim of AU detection methods is to 976 produce a binary frame-level label per target AU, indicating 977 whether the AU is active or not. Both AU intensity estima- 978 tion and temporal segment detection aim at inferring frame- 979 level labels of these concepts as described in the FACS man- 980 ual (see Section 2). AU classification was a problem targeted 981 early in the field, uncommon nowadays, and deals with 982 sequences containing pre-segmented AU activation epi- 983 sodes. The problem is then simplified to performing per- 984 sequence labelling.

AU problems are characterised by important temporal 986 and spatial correlations. Spatial correlations refer to the 987 well-known fact that some AUs tend to co-occur. Temporal 988 
correlations instead relate to the constraints resulting from the temporal nature of the data. However, most techniques capturing these correlations build on frame-level inference methods. Thus, we first review frame-based learning technique (Section 5.1), listing problem-specific approaches. We devote Section 5.2 to techniques that harness the temporal correlations in the output space derived from analysing video sequences. Methods that capture the so-called spatial relations are the subject of Section 5.3. Some techniques propose a single model capturing both spatial and temporal relations (Section 5.4). We further review some techniques that do not align with this taxonomy as they tackle complementary aspects, devoting a section to dimensionality reduction (Section 5.5), transfer learning (Section 5.6) and unsupervised learning of facial events (Section 5.7). A broad overview of different learning methodologies for AU analysis can be found in Fig. 3 in [43].

\subsection{Analysis of Individual AU}

Contemporary datasets are composed of video sequences, and we consider the analysis of still images to be a sub-optimal approach. In truly challenging data videos are not presegmented, so that the target AU can occur at any time in the video, or may not appear at all. Two approaches can be distinguished for detecting and temporally localising an AU: frame-level approaches and segment-level approaches.

Frame-level labelling methods perform inference at each frame of the sequence, assigning one of the target labels to each of them. However, labels obtained through frame-level inference typically result in temporally inconsistent label sequences (e.g., isolated single frames labelled as active are in all likelihood incorrect). Thus, a performance improvement can be attained by combining frame-level information with temporal consistency information, which is typically done through the use of graphical models.

Segment-based approaches focus instead on localising events as a whole, taking as input a representation of a spatio-temporal data segment. If this is deemed to be a positive instance, then each frame within it is assigned the associated label. This approach has an inherent mechanism for producing temporally-consistent predictions. Yet, segment-based approaches are uncommon, mostly due to the complex nature of this type of algorithms, and the challenge of representing video segments of variable length.

We start by describing how to deal with frame-level inference, considering the different AU-related problems in the literature. Then we describe different approaches for incorporating temporal consistency on the predicted labels. Finally, we describe works in segment-based learning.

Frame-based $A U$ detection aims to assign a binary label per target AU indicating activation to each of the frames in the sequence. Common binary classifiers applied to this problem include Artificial Neural Networks (ANN), Boosting techniques, and Support Vector Machines (SVM). ANNs were the most popular method in earlier works, e.g., [19], [53], [173]. However, ANNs are hard to optimise. While the scalability of ANN to large datasets is one of its strongest aspects, the amount of available data for AU analysis remains relatively scarce. It would nonetheless be interesting to study their performance given the recent resurgence of ANN, specially as some promising works have recently appeared [71], [81]. Boosting algorithms, 1049 such as AdaBoost and GentleBoost, have been a common 1050 choice for AU recognition, e.g., [73], [208]. Boosting algo- 1051 rithms are simple and quick to train. They have fewer 1052 parameters than SVM or ANN, and can be less prone to 1053 overfitting. They implicitly perform feature selection, 1054 which is desirable for handling high-dimensional data and 1055 speeding up inference, and can handle multiclass classifi- 1056 cation. However, SVM are nowadays the most popular 1057 choice, e.g., [31], [108], [202], [209]. SVMs provide good 1058 performance, can be non-linear, parameter optimisation is 1059 relatively easy, efficient implementations are readily avail- 1060 able (e.g., the libsvm library, [26]), and a choice of kernel 1061 functions provides extreme flexibility of design. 1062

AU Intensity Estimation. Estimating AU intensity is of 1063 interest due to its semantic value, allowing higher level 1064 interpretation of displayed behaviour for which the inten- 1065 sity of facial gesture is informative (e.g., discrimination 1066 between polite and joyful smiles). The goal in this scenario 1067 is to assign, for each target AU, a per-frame label represent- 1068 ing an integer value from 0 to 5. This problem can be 1069 approached using either a classification or a regression. $\quad 1070$

Some approaches use the confidence of a binary frame- 1071 based AU detection classifier to estimate AU intensity. The 1072 rationale is that the lower the intensity is, the harder classi- 1073 fying the example will be. For example, [15] used the dis- 1074 tance of the test sample to the SVM separating hyperplane, 1075 while [73] used the confidence of the decision given by Ada- 1076 Boost. It is however more natural to treat the problem as 6- 1077 class classification. For example, [108] employed six one- 1078 versus-all binary SVM classifiers. Alternatively, a single 1079 multi-class classifier (e.g., ANN or a Boosting variant) could 1080 be used. The extremely large class overlap means however 1081 that such approaches are unlikely to be optimal.

AU intensity estimation is nowadays most often posed as 1083 a regression problem. Regression methods penalise incorrect 1084 labelling proportionally to the difference between ground 1085 truth and prediction. Such structure of the label space is 1086 absent in the most common classification methods. The large 1087 overlap between classes also implies an underlying continu- 1088 ous nature of intensity that regression techniques are better 1089 equipped to model. Examples include Support Vector 1090 Regression, [86], [157], or Relevance Vector Regression so 1091 that a probabilistic prediction is obtained [90]. Furthermore, 1092 [69] shows performance comparisons between binary classi- 1093 fication-based, multi-class and regression-based intensity 1094 estimation, showing that the latter two attain comparable 1095 performance, but improve significantly over the former for 1096 the task of smile intensity estimation. An alternative is the 1097 use of Ordinal Regression. Ordinal regression maps the 1098 input feature into a one dimensional continuous space, and 1099 then finds some binning thresholds tasked with splitting the 1100 $n$ classes. During training, both the projection and the bin- 1101 ning values are estimated jointly [144].

\subsection{Temporal Consistency}

Temporal phase modelling. Temporal consistency can be 1104 enforced through the modelling and prediction of AU tempo- 1105 ral phases (neutral, onset, apex or offset) and their transitions 1106 (see Section 2 for their definition). It constitutes an analysis of 1107 the internal dynamics of an AU episode. Temporal phases 1108 


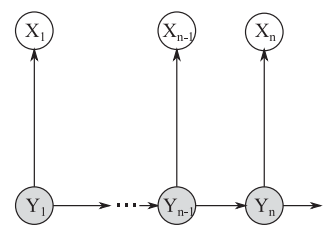

(a)

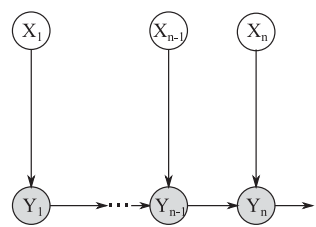

(b)

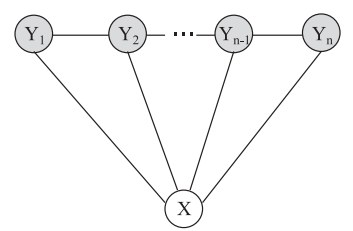

(c)

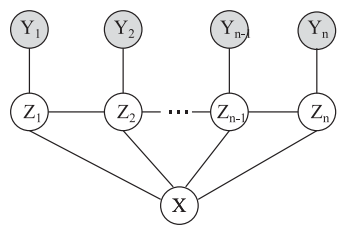

(d)

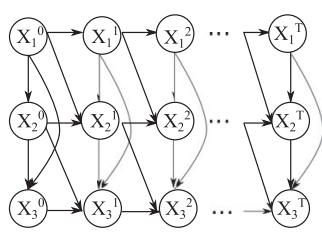

(e)

Fig. 8. Graphical illustration of (a) Hidden Markov Model, (b) Maximum entropy Markov model, (c) Conditional random field, (d) Hidden conditional random field, (e) Dynamic bayesian network. $\mathbf{X}$ is the observation sequences, $\mathbf{Z}$ is the hidden variables and $\mathbf{Y}$ is the class label.

add important information about an AU activation episode, as all labels should occur in a specific order.

Temporal segment detection is a multi-class problem, and is typically addressed by either using a multi-class classifier or by combining several binary classifiers. Early work used a set of heuristic rules per AU based on facial landmark locations [132]. More recent approaches use discriminative classifiers learnt from data. Among them, [191] uses one-versus-one binary SVMs (i.e., six classifiers) and a majority vote to decide on the label, while [88], [94] trained GentleBoost classifiers for each temporal segment ([94] excluded apex as it used motion-based features). These works use a score measure provided by the classifier to represent the confidence of the label assignments.

It is important to note however that reliably distinguishing the temporal segments based on the appearance of a single frame is impossible. Appearance relates to the AU intensity, and apex, onset or offset frames can be of practically any intensity. Temporal segments are characterised instead by the intensity evolution (i.e., its derivatives). Therefore, the use of temporal information is mandatory. The aforementioned works encode this information at the feature level and through the use of graphical models (see below).

Graph-based methods: In frame-based approaches, temporal consistency is typically enforced by employing a graphical model. Some methods divide the problem into two steps. First a frame-level ML method of choice is used to obtain soft perframe predictions, and then a (typically Markov chain) transition model is used to encode how likely each label change is. Then, the Viterbi decoding algorithm can be used to find the most likely sequence of predictions [88], [94], [187], [191]. This approach can be used irrespective of the problem targeted, and has for example been used for AU detection using the margin of an SVM classifier to perform the soft assignment [191], and for AU temporal segment detection using the probability yielded by a GentleBoost algorithm [88], [94]. This model is similar to an HMM, but a discriminative classifier substitutes the generative model relating data and labels. This results in the topology of the Maximum Entropy Markov model (MEMM, [116], see Fig. 8), where the classifier and the temporal consistency models are trained independently.

It can however be advantageous to jointly optimise the transition model and the frame-level classifier. For example, [114] propose to use a Hidden Markov Model for AU intensity estimation. Discriminative methods such as Conditional Random Fields (CRF) (see Fig. 8) might however be more effective [195]. CRF is an undirected graph, and the associated potentials are discriminatively trained. A chain CRF is its simplest topology. Each label node indicates the perframe output label. The state of the label node depends on the immediate future and past labels and on the data term.
CRFs restrict the frame-level learning algorithm to log-lin- 1160 ear models. Several extensions of CRF have been applied to 1161 AU-related problems, aiming to incorporate even more 1162 information in the model. For example, the kernel Condi- 1163 tional Ordinal Random Fields was applied to the AU tem- 1164 poral segment detection problem in [144], and makes use of 1165 the temporal ordering constraints of the labels. Another 1166 extension was proposed in [197], where the authors pro- 1167 posed a Latent CRF where the latent variables can switch 1168 between nominal to ordinal types. Instead, [27] proposed a 1169 modified version of the Hidden Conditional Random Field 1170 (HCRF, see Fig. 8). This model assumes known AU labels 1171 for the start and end frame. Observations provide evidence 1172 of AU activation (the hidden variables), while facial expres- 1173 sions are simultaneously inferred from the binary informa- 1174 tion on AU activations. In this way, the detection of AU and 1175 prototypical expressions is learnt jointly.

Most graphical models are trained by maximising the 1177 empirical log-likelihood. However, some AU-related prob- 1178 lems (specially AU intensity estimation) suffer greatly from 1179 label unbalance. Introducing label-specific weights on the 1180 loss function is complicated in this case, and models may suf- 1181 fer from a bias towards more common classes. The most 1182 immediate way to tackle this problem is to train a frame-level 1183 discriminative classifier beforehand using class weights, and 1184 to feed the output of this model to the graph (hence the suc- 1185 cess of the two-step approach). A more complex solution 1186 might involve using alternative graph formulations, e.g., 1187 Max-margin graphs [170].

Segment-based methods: Early datasets were composed of 1189 short (10-100 frames) pre-segmented sequences with well- 1190 defined AU activations. This particular case can be addressed 1191 by using a sequence classifier, for example an HMM (see 1192 Fig. 8). For example, [101] trained a different HMM per class. 1193 At test time, each HMM is evaluated and the class assigned is 1194 the one yielding the highest likelihood. Alternatively, all 1195 frames of the sequence can be analysed using a per-frame 1196 binary classifier (see Section 5.1), and a majority vote is cast to 1197 assign a sequence label [193]. However, the availability of 1198 pre-segmented AU episodes at test time is unrealistic in any 1199 practical scenario and nowadays this problem is basically 1200 discontinued.

Most segment-based methods deal instead with unseg- 1202 mented data, and the problem consists of finding the start- 1203 ing and end point to the event maximising a score. As 1204 opposed to frame-based methods, learning uses patterns 1205 representing the whole event at once. This is also different 1206 in nature to graph-based models, which typically relate 1207 data and labels through frame-level patterns. The need to 1208 describe segments of varying length through a feature of 1209 the same dimensionality imposes a strong restriction on the 1210 
possible data representations used. Furthermore, features should be robust against variations on the action temporal patterns such as the speed of execution. The output of segment-based methods consists of a single label for a whole section of the test sequence, but it can be directly translated into frame-level labelling.

One such approach was proposed by [164]. The authors proposed a segment-based classifier, coined KSeg-SVM, that uses a bag of temporal words to represent the segments. The structured-output SVM framework [179] is used for inference and learning, drawing a clear parallelism with the work in [21]. Alternatively, [51] proposed to combine frame-level with segment-level methodologies in what they call a cascade of classifiers. They show that the use of segment information in a step subsequent to frame-based inference leads to better performance. While these methods are compared against frame-level equivalents, the authors omit a comparison with graph-based models, which constitutes the most logical alternative.

An alternative problem formulation is that of performing weakly-supervised learning. In this scenario, training instances are sequences, and the labels indicate whether an AU occur within the sequence but without indicating where exactly. This problem was considered by [171], where a Multiple Instance Learning (MIL) approach was used to tackle AU detection. A similar problem was tackled in [145], where the authors propose a new MIL framework to deal with multiple high-level behaviour labels. The interest in these techniques stems from the ease of manual sequencebased annotation, and from its use for problems where labelling is more subjective.

\subsection{Spatial Relations}

It is well-known that some AUs frequently co-occur (see Section 2). Thus, it is only natural to exploit these correlations and to perform joint inference of multiple AUs. In here we distinguish between methods that exploit correlations by learning a joint feature representation, and methods that impose correlations among labels, typically by employing graphs. Finally, temporal correlations can also be taken into account to extend frame-level models, thus incorporating both cooccurrence and temporal consistency correlations.

Joint representation: The early seminal work by [172] already exploited the flexibility of ANN, defining the output layer to have multi-dimensional output units. Each output can fire independently, indicating presence of a specific AU, but all AUs share an intermediate representation of the data (the values on the hidden layer). More recently, [229] used a Multi-Task Feature Learning technique to exploit commonalities in the representation of multiple AUs. The same strategy was followed by [217], but in this case the tasks are organised in a hierarchical manner, with AU at the leaf nodes and groups of AU at higher levels (the hierarchy is hand-crafted rather than data driven).

Label-space correlations: Graphical models can be employed in a similar manner as for temporally-structured inference. However, the graph topology in the latter case arise more naturally from the temporal ordering. In this case, which AU correlations are considered by the topology will result in different performances, and there is no standard way of selecting them. Approaches include [177], which proposed to use a directed graph, Bayesian networks (BN). BN capture 1271 pairwise correlations between AUs, do not need to explicitly 1272 select the AU correlations to be modelled, and they can scale 1273 to a large number of correlations. Alternatively, [150] pre- 1274 sented a methodology for joint AU intensity estimation based 1275 on Markov random fields (MRF). First, frame-based regres- 1276 sion models were trained for each AU, and their outputs were 1277 used as inputs to a MRF with pairwise potentials. Since MRF 1278 is an undirected graph, the topology is restricted to a tree 1279 structure to achieve fast and exact inference. Loopy graphs 1280 could be used too, but then they would require approximate 1281 inference, and thus it is unclear whether it would result in a 1282 performance gain. Several different hand-crafted topologies 1283 were evaluated.

While capturing pairwise relations can significantly 1285 improve performance, some of the relations involve larger 1286 sets of AU. For example, some AUs are connected due to 1287 their co-occurrence in frequently occurring facial expres- 1288 sions (e.g., AU6 and AU12 in smiles). Thus, capturing 1289 higher-order relations (beyond pairwise) can yield further 1290 benefits. One such model was proposed in [198], where a 1291 variant of Restricted Boltzmann Machines (RBM, [77]) was 1292 used to capture more complex relations, and to jointly incor- 1293 porate reasoning regarding prototypical facial expressions. 1294 Instead, [146] proposed to combine the learning of AU and 1295 facial expressions together. Prior knowledge of the correla- 1296 tions between AU and expressions (found through manual 1297 labelling) are also incorporated. A hierarchical approach 1298 was followed in [91], which greedily constructed a genera- 1299 tive tree with labels and features at the leaf nodes. Each 1300 node on the upper layer joins a pair of lower-level nodes. 1301 The resulting trees are used to perform AU intensity estima- 1302 tion. Finally, [166] employed a graphical model, a variant of 1303 the Bayesian compressed sensing framework, capable of 1304 grouping AU (where an AU can be on more than one 1305 group), and imposing sparsity so few AU can be active at a 1306 time. While this captures correlations beyond pairwise, they 1307 need to resort to complex variational inference.

An alternative encoding which avoids the use of graphi- 1309 cal models was proposed in [223]. Label correlations were 1310 imposed in a discriminative framework. Regularisation 1311 terms for each of the AU pairs considered were introduced 1312 in the learning loss function, penalising (dis)agreement 1313 between correlated AUs.

\subsection{Spatio-Temporal Relations}

Capturing both spatial and temporal correlations has the 1316 potential for further performance benefits. Factors such as 1317 facial expressions, head or body movements and poses, or 1318 higher-level interpretations of the data, can also be inte- 1319 grated into a single inference framework. If directed graphs 1320 are used, the complexity of the inference grows very quickly 1321 due to the appearance of loops in graphs, leading to approx- 1322 imate inference and a potential performane loss. It is thus 1323 only natural that works within this category focus on 1324 directed graphs.

Existing efforts include [177], where temporal correlations 1326 were captured by means of a Dynamic Bayesian Network 1327 (DBN). DBNs extend BNs by incorporating temporal infor- 1328 mation, with each time slice of a DBN being a BN. Similarly, 1329 DBNs extend HMMs by being able to handle multiple 1330 
interacting variables at a given time frame. Therefore, this model combines both the temporal correlations of HMM-like methods, and the joint AU estimation of BN. A further extension was presented in [176], where the authors integrate "non-AU" factors, such as head pose, into a joint probabilistic model. The same approach was followed by [99], but in this case the DBN was applied to perform AU intensity estimation. One-vs-one SVMs were used as input to the DBN.

\subsection{Dimensionality Reduction}

Due to the typically high dimensionality of the input features, it is often recommended (but not strictly necessary) to reduce the input dimensionality prior to the application of other learning techniques. This can be done through feature selection, manifold learning or pooling. Feature selection aims to find a subset of the original features that are representative enough, and it is typically a supervised approach. Manifold learning methods, such as PCA, find underlying lower-dimensional structures that preserve the relevant information from the original data. Pooling combines features from neighbouring (spatial) locations into a single feature, for example by computing their average or their maximum. These techniques have been well covered in a recent survey on facial AU analysis, and we refer the reviewer to it for further discussion [154].

\subsection{Transfer Learning}

One of the important aspects of AU-related data is that nuisance factors can greatly affect AU representation and thus hinder the generalisation capability of the models learnt. One way of dealing with this problem is to use transfer learning or domain adaptation. These are most commonly applied when there is a significant difference between the distribution of the training data and the test data, so that models learnt on the training data (e.g., containing frontal head pose videos only) might be sub-optimal for the test data (e.g., presenting multiple head poses).

Transfer learning encompasses a wide range of techniques designed to deal with these cases [129]. In the transfer learning literature, inductive learning refers to the case where labelled data of the target domain (where we want to apply the learnt methods) is available. Transductive learning makes no such assumption, with the target domain data being purely unsupervised [129]. Transfer learning has only very recently been applied to automatic AU analysis. For example, [33] proposed a new transductive learning method, referred to as Selective Transfer Machine (STM). Because of its transductive nature, no labels are required for the test subject. At test time, a weight for each training example is computed as to maximise the match between the weighted distribution of training examples and the test distribution. Inference is then performed using the weighted distribution. The authors obtained a remarkable performance increase, beating subject-specific models. However, reduced availability of subject-specific training examples might partially explain this. [152] and [212] proposed a discriminative regression method tasked with predicting subject-specific model parameters. The input consisted of the distribution of frame-level features corresponding to the subject (e.g., extracted from a video), and different measures for comparing distributions are studied. Instead, [213] decoupled the problem of AU detection into the detection 1390 for easy and hard frames. The easy detector provides a set 1391 of confident detections on easy frames, which are then used 1392 to adapt a second classifier to the specific test-time subject 1393 in order to facilitate the finder-grained detection task. 1394

In contrast, [29] evaluated standard methodologies for 1395 both inductive and transductive transfer learning for AU 1396 detection, finding that inductive learning improved the per- 1397 formance significantly while the transductive algorithm led 1398 to poor performance. Multi-task learning (MTL) can also be 1399 used to produce person-specific AU models. For example, 1400 [143] proposed an inductive tensor-based feature learning 1401 MTL method simultaneously capturing correlations among 1402 AU and correlations among subjects. Alternatively, [3] built 1403 upon a MTL algorithm capable of estimating tasks related- 1404 ness. The task relations were designed to encode subject 1405 similarity, being thus shared across AU, and AU-specific 1406 dictionaries translating these latent relations into model 1407 parameters were learnt. Current Deep Learning methodolo- 1408 gies rely systematically on transfer learning, typically using 1409 ImageNet pre-trained models and typically fine-tuning the 1410 models to the task at hand. Features at lower layers are 1411 shown to be of general applicability and well-posed for 1412 transfer to other tasks. This allows successful training with 1413 much less training data. See Section 4.4 for further discus- 1414 sion on deep learning for AU analysis.

Transfer learning is a promising approach when it comes 1416 to AU analysis. Appearance variation due to identity are 1417 often larger than expression-related variations. This is aggra- 1418 vated by the high cost of AU annotation and the low number 1419 of subjects in datasets. Therefore, techniques that can capture 1420 subject-specific knowledge and transfer it at test time to 1421 unseen subjects are highly suitable for AU analysis.

\subsection{Unsupervised Discovery of Facial Events}

In order to overcome the scarcity of training data, which 1424 impedes development of robust and highly effective 1425 approaches to machine analysis of AUs, some recent efforts 1426 focus on unsupervised approaches. The aim is in this case 1427 to segment a previously unsegmented input sequence into 1428 relevant facial events, but without the use of labels during 1429 training [49], [224]. The facial events might not be coincident 1430 with AU, although some correlation with them is to be 1431 expected, as AUs are distinctive spatio-temporal events. 1432 Existing works apply a sequence-based clustering algorithm 1433 to group events of similar characteristics. For example, [224] 1434 used a dynamic time alignment kernel to compare sub- 1435 sequences in a manner invariant to the speed of the facial 1436 action. Instead, [210] used Slow Feature Analysis to learn, in 1437 an unsupervised manner, a latent space that correlates with 1438 the AU temporal segments. In this case, a quantitative per- 1439 formance evaluation of this correlation was provided. 1440 Despite its interesting theoretical aspects, the practical 1441 applicability of purely unsupervised learning is not clear. A 1442 semi-supervised learning setting [28], [215] might result in a 1443 more sensible approach, as it uses all the annotated data 1444 together with potentially useful unannotated data. Such an 1445 approach is not immediate and has not been explored yet. 1446 Finally, [34] proposed an unsupervised methodology for, 1447 given two or more video streams containing persons inter- 1448 acting, detecting events of synchrony between the subjects, 1449 


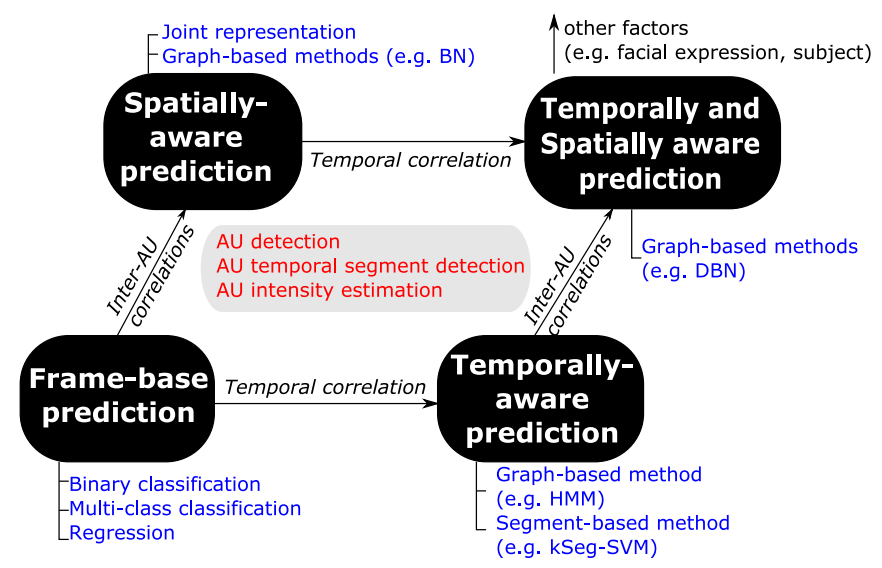

Fig. 9. Relations between some of the methodologies. Arrows indicate relations in terms of the output correlations considered. Nodes indicate a grouping of methodologies considering the same output correlations. Sections containing works within a category are shown in green.

understood as overlapping segments of the video where the subjects present similar facial behaviour. Another interesting discussion on the topic, including references to similar works on different domains, can be found in [96].

\subsection{Discussion}

What model works best?: Techniques requiring little training data are still useful for AU problems. The scarcity of data means that high-capacity models, with more flexible kernels, hidden layers or model variables might not necessary perform better. Using the temporal and spatial structure of the problem is more likely to yield a performance gain. A graphical depiction of the relations between different methods depending on the correlations considered is shown in Fig. 9. Moving in any direction on the graph shown adds (or removes) a new source of correlations. We further sketch a third dimension: the correlation with "non-AU" information. Performing an adequate feature fusion strategy can also yield solid performance. Models capable of creating personalised models are very interesting, although they are at an early stage of research.

How can correlations be used in practice?: The most effective and studied way is to use graphs. Temporal correlations are easy to obtain and provide important performance improvements. Due to severe label imbalance, it is a good idea to pre-train your (typically discriminative) frame-based model of choice, and then use a graphical model taking the output confidence as the input to the graph.

Why not include everything in one graph?: This approach was the one followed by [176], although they were restricted to using directed graphs. Instead, adding spatial and temporal correlations together in an undirected graph can lead to loops. Loopy graphs result in slow and approximate inference. How to include all of this information into an undirected graph and yet attain fast and exact solution (or even a good approximation) is not clear. Thus, more complex graphs do not necessarily lead to better performances.

Opportunities and directions An important direction of research is the aforementioned problem of how to incorporate more information in graphs without resorting to slow and approximate inference. Furthermore, transfer learning and domain adaptation are well suited to AU-related problems, and are very relevant nowadays in the CV and ML 1491 fields in general. Temporal models are often restricted to 1492 Markov chains. This might result in a lot of missing temporal 1493 correlations, and non-Markov (e.g., multi-scale) models 1494 could be of use. However, temporal patterns might be 1495 domain dependent and much more data would be needed to 1496 obtain models generalisable to unseen test conditions. 1497 Graphs capturing higher-order correlations (involving more 1498 than two nodes), or the design of discriminative graphs capa- 1499 ble of handling data imbalance, could be interesting steps too. 1500

Combining ML models: Given the subtle signals that AU 1501 analysis depends on, and given the low number of training 1502 examples available, the use of specialized ML models focus- 1503 ing on easier, better-posed problems seems like a natural 1504 research direction. For example, [51] used frame-level, 1505 segment-level and onset/offset detector models in combina- 1506 tion (a similar approach was successfully proposed for 1507 facial expression recognition in [47]). Alternatively, other 1508 methods focused on combining ML models trained to 1509 respond to specific parts of the face, e.g., [80], [103]. In this 1510 way, the spatially localized nature of AUs can be exploited, 1511 and the features used for learning contain less variation 1512 than when encoding the whole face.

\section{Data AND Databases}

The need for large, AU labelled, publicly available data- 1515 bases for training, evaluating and benchmarking has been 1516 widely acknowledged, and a number of efforts to address 1517 this need have been made. In principle, any facial expression 1518 database can be extended with AU annotation. However, 1519 due to the very time-consuming annotation process, only a 1520 limited number of facial expression databases are FACS 1521 annotated, and even fewer are publicly available. They can 1522 be divided into three groups: Posed facial expression data- 1523 bases, spontaneous facial expression databases and 3D facial 1524 expression databases. Although the scope of this survey is 1525 restricted to automatic 2D AU analysis, 3D databases enable 1526 the rendering of 2D examples in arbitrary head poses. 1527

For completeness, we provide a summary of existing 1528 facial AU-annotated databases in Table 3. However, a more 1529 in-depth coverage of this topic can be found in [43].

\subsection{Training Set Selection}

The choice of training examples is a relatively neglected 1532 problem when it comes to automatic AU analysis. Most of 1533 the existing works use one of two simple approaches. One 1534 approach assigns fully expressive frames to the positive 1535 class and frames associated with other AUs to the negative 1536 class. This approach maximises the differences between 1537 positive and negative classes, but results in a large imbal- 1538 ance between them, especially for infrequent AUs [228]. In 1539 this case, peak frames may provide too little variability to 1540 achieve good generalisation, and faces with active but not 1541 fully expressive AUs might have patterns unseen in the 1542 training set. The other approach reduces imbalance between 1543 classes by including all target frames from onset to offset in 1544 the positive class (e.g., [31], [161], [66]). However, because 1545 frames near the beginning of the onset and the end of the 1546 offset phases often differ little from neutral ones, separabil- 1547 ity of classes is compromised and the number of false posi- 1548 tives might increase accordingly. 
TABLE 3

FACS-Annotated Facial Expression Databases

\begin{tabular}{|c|c|c|c|c|c|c|c|}
\hline Database & Elicitation method & Size & Camera View & $\mathrm{S} / \mathrm{D}$ & Act & oao & Int \\
\hline$\overline{\text { AM-FED [117] }}$ & Induced & $\mathrm{N} / \mathrm{A}$ & Various head poses & $\mathrm{D}$ & 10 & $\mathrm{~N}$ & $\mathrm{~N}$ \\
\hline Bosphorous [155] & On command & 105 subjects & 3D multi-pose & $S$ & 25 & $\mathrm{~N}$ & $\mathrm{Y}$ \\
\hline BP4D [218] & Induced & 41 subjects & 3D multi-pose & $\mathrm{D}$ & 27 & $\mathrm{~N}$ & $\mathrm{Y}$ \\
\hline CASME [207] & Induced (micro) & 35 subjects & Near frontal & $\mathrm{D}$ & $\mathrm{F}$ & Y & $\mathrm{N}$ \\
\hline CASME II [206] & Induced (micro) & 26 subjects & Near frontal & $\mathrm{D}$ & $\mathrm{F}$ & $\mathrm{Y}$ & $\mathrm{N}$ \\
\hline Cohn-Kanade [92] & On command & 97 subjects & Frontal & $\mathrm{D}$ & $\mathrm{F}$ & $\mathrm{Y}$ & $\mathrm{N}$ \\
\hline Cohn-Kanade+ [105] & Naturally occurring & 26 subjects & Frontal \& $15^{\circ}$ side view & $\mathrm{D}$ & 8 & $\mathrm{~N}$ & $\mathrm{~N}$ \\
\hline D3DFACS [167] & On command & 10 subjects & 3D multi-pose & D & $\mathrm{F}$ & $\mathrm{N}$ & $\mathrm{N}$ \\
\hline DISFA [115] & Induced & 27 subjects & Near-frontal & $\mathrm{D}$ & 12 & $\mathrm{~N}$ & $\mathrm{Y}$ \\
\hline GEMEP-FERA [188] & Acted & 10 subjects & Significant head movement & $\mathrm{D}$ & 12 & $\mathrm{~N}$ & $\mathrm{~N}$ \\
\hline ICT-3DRFE [44] & On command & 23 subjects & 3D multi-pose & $S$ & $\mathrm{~F}$ & $\mathrm{~N}$ & $\mathrm{Y}$ \\
\hline MMI (Part I-III) [135] & On command & 210 subjects & Frontal \& Profile & SD & $\mathrm{F}$ & $\mathrm{Y}$ & $\mathrm{N}$ \\
\hline MMI (Part IV-V) [190] & Induced & 25 subjects & Frontal & $\mathrm{D}$ & $\mathrm{F}$ & $\mathrm{N}$ & $\mathrm{N}$ \\
\hline ISL Frontal [177] & On command & 10 subjects & Near frontal & $\mathrm{D}$ & 14 & Y & $\mathrm{N}$ \\
\hline ISL Multi-view [176] & On command & 8 subjects & Frontal, $15^{\circ} \& 30^{\circ}$ side & $\mathrm{D}$ & 15 & $\mathrm{Y}$ & $\mathrm{N}$ \\
\hline Sayette GFT [68] & Naturally occurring & 96 subjects & Frontal & $\mathrm{D}$ & 20 & $\mathrm{~N}$ & $\mathrm{Y}$ \\
\hline SEMAINE [118] & Induced & 150 subjects & Frontal \& Profile & $\mathrm{D}$ & 6 & $\mathrm{~N}$ & $\mathrm{~N}$ \\
\hline UNBC-McMaster [107] & Induced(Pain) & 129 subjects & Frontal & $\mathrm{D}$ & 10 & $\mathrm{~N}$ & $\mathrm{Y}$ \\
\hline
\end{tabular}

Elicitation method: On command/Acted/Induced/Interview. Size: number of subjects. Camera view: frontal/profile/3D. S/D: static (image) or dynamic (video) data. Act: AU activation annotation (number of AUs annotated, F-fully annotated). oao: onset/apex/offset annotation. Int: intensity (A/B/C/D/E) annotation.

Apart from these standard approaches, [88] proposed a heuristic approach for training example selection. They take the first apex frame of each target $\mathrm{AU}$, plus any apex frames where any other AUs are active independently of its current temporal phase. The idea is that appearances of AU combinations are different than those of AUs happening in isolation, so they should be properly represented on the training set. However, in order to avoid repetitive patterns, the training set only includes one frame where all AUs are in their apex phase. An adapted version of this heuristic was used in [188], as no annotations of the temporal segments were available. [88] also defines a different heuristic to extract dynamic appearance features. They first define salient moments, to wit, the transition times between the different temporal segments and the midpoint of every AU phase. Then a temporal window centred at these points is used to compute the training patterns.

Zhu et al. [228] propose dynamic cascades with bidirectional bootstrapping, which combines an Adaboost classifier with a bootstrapping strategy for both positive and negative examples. Wrongly classified negative examples are re-introduced in the training set, and the set of positives is enhanced with less obvious examples correctly detected by the classifier (what the authors call spreading). The classifier is then retrained, leading to an iterative procedure that is repeated until convergence.

\subsection{Discussion}

While researchers now have a much wider range of $\mathrm{AU}$ annotated databases at their disposal than 10 years ago, when basically only the Cohn-Kanade and MMI databases were available [92], [190], lack of high-quality data remains a major issue. Recent advances in statistical machine learning such as CNNs require data volumes orders in magnitude larger than currently available. In addition, there is an issue with the reliability of manual AU labelling in a number of databases. While FACS is touted to be an objective human measurement system, there remain subjective interpretations, and the quality of labelling is highly depen- 1587 dent on the amount of experience a FACS annotator has. 1588 Ideally, the inter-rater reliability of AU annotation should 1589 be reported for each database.

Another issue relates to ethical considerations. Some 1591 excellent spontaneous facial action databases are not pub- 1592 licly available due to human-use considerations (e.g., [2], 1593 [37], [159]). In general, many contemporary issues for which 1594 automatic AU detection would be a great benefit (e.g., auto- 1595 matic analysis of depression or other medical conditions) 1596 will use that that is hard to share with other researchers. 1597 These datasets represent a potentially valuable trove of 1598 training and testing data. Developing methods to allow 1599 other researchers benefit from these data without having 1600 direct access to them would greatly benefit the community. 1601

\section{Challenges and Opportunities}

Although the main focus in machine analysis of AUs has 1603 shifted to the analysis of spontaneous expressions, state-of- 1604 the-art methods cannot be used in fully unconstrained envi- 1605 ronmental conditions effectively. Challenges preventing 1606 this include handling occlusions, non-frontal head poses, 1607 co-occurring AUs and speech, varying illumination condi- 1608 tions, and the detection of low intensity AUs. Lack of data is 1609 another nagging factor impeding progress in the field.

Non-frontal head poses occur frequently in naturalistic 1611 settings. Due to the scarceness of annotated data, building 1612 view-specific appearance-based approaches for automatic 1613 AU analysis is impractical. The existence of 3D databases 1614 may ease this problem, although rendering examples of 1615 AU activations at multiple poses is challenging as it 1616 involves simulating realistic photometric variance. Using 1617 head-pose-normalised images for learning and inference is 1618 a more feasible alternative. However, many challenges are 1619 associated with this approach. For example, the learning 1620 algorithms should be able to cope with partially corrupted 1621 data resulting from self-occlusions. More importantly, 1622 
TABLE 4

Performance on the FERA 2017 Challenge Benchmark Dataset

\begin{tabular}{lcc}
\hline Team & Occurrence detection & Intensity estimation \\
\hline Amirian et al. [8] & - & 0.295 \\
Batista et al. [18] & 0.506 & 0.399 \\
He et al. [75] & 0.507 & - \\
Li et al. [98] & 0.495 & - \\
Tang et al. [169] & $\mathbf{0 . 5 7 4}$ & - \\
Zhou et al. [225] & - & $\mathbf{0 . 4 4 5}$ \\
Baseline [184] & 0.452 & 0.217 \\
\hline
\end{tabular}

Occurrence performance is measured in terms of F1, and intensity in terms of ICC (see [184] for details).

head-pose normalisation while preserving facial expression changes is still an open problem that needs to be addressed.

Because AUs cause only local appearance changes, even a partial occlusion of the face can be problematic. So far, very limited attention has been devoted to this problem [102]. A possible solution is to rely on the semantics of AUs so that occluded AUs can be inferred from the visible ones or from models of AU temporal co-occurrence and consistency.

It is rare that AUs appear in isolation during spontaneous facial behaviour. In particular, the co-occurrences of AUs become much harder to model in the presence of nonadditive AUs (see Section 2). Treating these combinations as new independent classes [109] is impractical given the number of such non-additive AU combinations. On the other hand, when treating each AU as a single class, the presence of non-additive combinations of AUs increases the intraclass variability, potentially reducing the performance [88]. Also, the limited number of co-occurrence examples in existing AU-coded databases makes this problem really difficult. Hence, the only way forward is by means of modelling the "semantics" of facial behaviour, i.e., temporal cooccurrences of AUs. This is an open problem that has not received proper attention from the research community. Beyond data-driven approaches, it is a well-known anatomical fact that some AU cannot co-occur together. Incorporating this domain knowledge can help constrain the problem further [198]. An interesting associated problem is learning with annotations of a subset of AU [201], as most datasets annotate different AU subsets.

While the importance of facial intensities and facial dynamics for the interpretation of facial behaviour has been stressed in the field of psychology (e.g., [65], [5]), it has received limited attention from the computer science community. The detection of AU temporal segments and the estimation of their intensities are unsolved problems. There is some degree of class overlap due to unavoidable labeller noise and unclear specifications of the class boundaries. Clearer annotation criteria to label intensity in a continuous real-valued scale may alleviate this issue. Building tools to improve performance in the presence of inter-labeller disagreement is therefore important.

All AU-coded databases suffer from various limitations, the most important being the lack of realistic illumination conditions and naturalistic head movements. This might mean that the field is driving itself into algorithmic local maxima [199]. Creating publicly available "in-the-wild" dataset is therefore of importance.
The absence of an adequate benchmark dataset has also 1671 been a detrimental factor for the evolution of the field. The 1672 facial expression and analysis challenge (FERA), organised 1673 in 2011, was the very first attempt [188], [189] to address 1674 this. A protocol was set in [188] where the training and test- 1675 ing sets were pre-defined and a performance metric was 1676 defined. This was followed by the FERA 2015 [186] and 1677 2017 [184] challenges, focussing on intensity estimation and 1678 AU detection under varying head-pose. The performance of 1679 the participants for FERA 2017 is shown in Table 4. 1680 Researchers can continue to submit their systems for evalu- 1681 ation on FERA 2017 to the organisers, who will update their 1682 website with new scores for as long as that remains relevant. 1683 The extended CK+ database has a similar function [105]. 1684 Reporting performance of proposed methodologies on these 1685 databases should be encouraged and other benchmarks 1686 with different properties are needed. Furthermore, the 1687 inclusion of cross-database experiments in the benchmark- 1688 ing protocol should be considered.

While many papers do report performance measures on 1690 publicly available datasets, this does not necessarily lead 1691 to a true comparison between methods. The way in which 1692 systems are trained and evaluated can differ significantly, 1693 leading to incomparable results. FERA and CK+ have 1694 helped somewhat by providing detailed evaluation proce- 1695 dures, but both datasets suffer from limited size and/or 1696 non-spontaneous expressions. Finally, the issue of unbal- 1697 anced data makes comparisons harder even further, as 1698 detailed by [84]. For all the above reasons, this survey 1699 does not include a quantitative performance comparison 1700 of existing systems.

Building personalised models using online and transfer 1702 learning methodologies ([33], [29]) is the way forward in 1703 our opinion. This is due to several reasons, as the lack of 1704 training data, the large subject differences, and the depen- 1705 dency of the displayed expressions on a large number of 1706 factors such as the environment, the task or the mood, 1707 which would be hard to cover exhaustively even if much 1708 larger amount of training data was available.

Low intensity AUs might be of special importance for sit- 1710 uations where the subject is intentionally controlling his 1711 facial behaviour. Scenarios as deceit detection would benefit 1712 greatly from the detection of subtle facial movements. The 1713 first research question relates to features that capture such 1714 changes [139].

Existing work deals mostly with classification or process- 1716 ing of the currently observed facial expressive behaviour. 1717 Being able to predict the subject's future behaviour given 1718 the current observations would be of major interest. This is 1719 a novel problem that can be seen as a long-term aim in the 1720 field. It is closely related to the already mentioned problem 1721 of modelling the semantics of AUs (facial behaviour) and 1722 should be studied in conjunction with it.

An interesting variant to the problem of AU detection 1724 was proposed in [141]. The authors propose to predict facial 1725 AU, but solely based on acoustic information. The authors 1726 use a Recurrent Neural Network to effectively capture tem- 1727 poral information, and test their models on a subset of the 1728 GEMEP database. This is an interesting idea, and opens up 1729 the possibility of tackling the AU problem from the audio- 1730 visual fusion perspective. 
Another interesting problem relates to the use of nonRGB modalities to either attain AU recognition, or to aid RGB-based AU recognition. For example, [82] performs AU recognition from thermal imagery by capturing differences in temperature related to muscle activation. Similarly, audio information can complement RGB-based recognition by distinguishing some sound-related expressions, like blowing or laughter. Depth information obtained from structured light or time of flight sensors forms another obvious opportunity for non-RGB based AU detection. Databases for analysis of this are now starting to come out [219].

Overall, although a major progress in machine recognition of AUs has been made over the past years, this field of research is still underdeveloped and many problems are still open waiting to be researched. Attaining a fully automatic and real-time AU recognition system capable of dealing with unconstrained environmental conditions would open up tremendous potential for new applications in games, security, and health industries and investing in this filed is therefore worthy all the effort. We hope that this survey will provide a set of helpful guidelines to all those carrying out the research in the field now and in the future.

\section{ACKNOWLEDGMENTS}

This work was funded by the European Union's Horizon 2020 research and innovation programme under grant agreement no 645378 .

\section{REFERENCES}

[1] N. Ahmed, T. Natarajan, and K. R. Rao, "Discrete cosine transform," IEEE Trans. Comput., vol. C-23, no. 1, pp. 90-93, Jan. 1974.

[2] S. Alghowinem, et al., "From joyous to clinically depressed: Mood detection using spontaneous speech," in Proc. 25th Int. Florida Artif. Intell. Res. Soc. Conf., 2012, pp. 141-146.

[3] T. Almaev, B. Martinez, and M. F. Valstar, "Learning to transfer: Transferring latent task structures and its application to personspecific facial action unit detection," in Proc. IEEE Int. Conf. Comput. Vis., 2015, pp. 3774-3782.

[4] T. Almaev and M. Valstar, "Local Gabor binary patterns from three orthogonal planes for automatic facial expression recognition," in Proc. Humaine Assoc. Conf. Affective Comput. Intell. Interaction, 2013, pp. 356-361.

[5] Z. Ambadar, J. F. Cohn, and L. I. Reed, "All smiles are not created equal: Morphology and timing of smiles perceived as amused, polite, and embarrassed/nervous," J. Nonverbal Behavior, vol. 33, pp. 17-34, 2009.

[6] Z. Ambadar, J. W. Schooler, and J. F. Cohn, "Deciphering the enigmatic face: The importance of facial dynamics in interpreting subtle facial expressions," Psychological Sci., vol. 16, no. 5, pp. 403-410, 2005.

[7] N. Ambady and R. Rosenthal, "Thin slices of expressive behavior as predictors of interpersonal consequences: A meta-analysis," Psychol. Bulletin, vol. 111, no. 2, pp. 256-274, 1992.

[8] M. Amirian, M. Kächele, F. Schwenker, and G. Palm, "Support vector regression of sparse dictionary-based features for viewindependent action unit intensity estimation," in Proc. IEEE Int. Conf. Autom. Face Gesture Recognit., 2017, pp. 854-859.

[9] A. B. Ashraf, S. Lucey, and T. Chen, "Reinterpreting the application of Gabor filters as a manipulation of the margin in linear support vector machines," IEEE Trans. Pattern Anal. Mach. Intell., vol. 32, no. 7, pp. 1335-1341, Jul. 2010.

[10] A. B. Ashraf, et al., "The painful face - pain expression recognition using active appearance models," Image Vis. Comput., vol. 27 , no. 12, pp. 1788-1796, 2009.

[11] A. Asthana, S. Zafeiriou, S. Cheng, and M. Pantic, "Incremental face alignment in the wild," in Proc. IEEE Conf. Comput. Vis. Pattern Recognit., 2014, pp. 1859-1866.
[12] A. Asthana, S. Zafeiriou, G. Tzimiropoulos, S. Cheng, and 1798 M. Pantic, "From pixels to response maps: Discriminative image 1799 filtering for face alignment in the wild," IEEE Trans. Pattern Anal. 1800 Mach. Intell., vol. 37, no. 6, pp. 1312-1320, Jun. 2015.

[13] T. Baltrušaitis, M. Mahmoud, and P. Robinson, "Cross-dataset 1802 learning and person-specific normalisation for automatic action 1803 unit detection," in Proc. 11th IEEE Int. Conf. Autom. Face Gesture 1804 Recognit. Workshop, 2015, pp. 1-6.

[14] T. Baltrušaitis, P. Robinson, and L.-P. Morency, “Openface: An 1806 open source facial behavior analysis toolkit," in Proc. IEEE Winter 1807 Conf. Appl. Comput. Vis., 2016, pp. 1-10.

[15] M. Bartlett, G. Littlewort, M. Frank, C. Lainscsek, I. Fasel, and 1809 J. Movellan, "Automatic recognition of facial actions in spontane- 1810 ous expressions," J. Multimedia, vol. 1, no. 6, pp. 22-35, 2006.

[16] M. S. Bartlett, J. C. Hager, P. Ekman, and T. J. Sejnowski, 1812 "Measuring facial expressions by computer image analysis," Psy- 1813 chophysiology, vol. 36, no. 2, pp. 253-263, 1999.

[17] M. S. Bartlett, et al., "Classifying facial actions," in Proc. Advances 1815 Neural Inform. Process. Syst., 1996, pp. 823-829.

[18] J. Batista, V. Albiero, O. Bellon, and L. Silva, "AUMPNet: Simulta- 1817 neous action units detection and intensity estimation on multipose 1818 facial images using a single convolutional neural network," in Proc. 1819 12th Int. Conf. Autom. Face Gesture Recognit., 2017, pp. 866-871.

[19] J. Bazzo and M. Lamar, "Recognizing facial actions using Gabor 1821 wavelets with neutral face average difference," in Proc. Int. Conf. 1822 Autom. Face Gesture Recognit., 2004, pp. 505-510.

[20] P. N. Belhumeur, D. W. Jacobs, D. J. Kriegman, and N. Kumar, 1824 "Localizing parts of faces using a consensus of exemplars," IEEE 1825 Trans. Pattern Anal. Mach. Intell., vol. 35, no. 12, pp. 2930-2940, 1826 Dec. 2013.

[21] M. B. Blaschko and C. H. Lampert, "Learning to localize objects 182 with structured output regression," in Proc. Eur. Conf. Comput. 1829 Vis., 2008, pp. 2-15.

[22] A. F. Bobick and J. W. Davis, "The recognition of human move- 1831 ment using temporal templates," IEEE Trans. Pattern Anal. Mach. 1832 Intell., vol. 23, no. 3, pp. 257-267, Mar. 2001.

[23] X. P. Burgos-Artizzu, P. Perona, and P. Dollár, "Robust face land- 183 mark estimation under occlusion," in Proc. IEEE Int. Conf. Com- 1835 put. Vis., 2013, pp. 1513-1520.

[24] X. Cao, Y. Wei, F. Wen, and J. Sun, "Face alignment by explicit 1837 shape regression," in Proc. IEEE Int. Conf. Comput. Vis. Pattern 1838 Recognit., 2012, pp. 2887-2894.

[25] X Cao, Y. Wei, F. Wen, and J Sun "Face alionment by explicit 1840 shape regression," Int. J. Comput. Vision, vol. 107, no. 2, pp. 177- 1841 190, 2014

[26] C. Chang and C. Lin, "LIBSVM: A library for support vector 1843 machines," ACM Trans. Intell. Syst. Technol., vol. 2, no. 3, 1844 pp. 27:1-27:27, 2011. Software Available: http://www.csie.ntu. 1845 edu.tw/ cjlin/libsvm

1846

[27] K. Chang, T. Liu, and S. Lai, "Learning partially-observed hidden 1847 conditional random fields for facial expression recognition," in 1848 IEEE Int. Conf. Comput. Vis. Pattern Recognit., 2009, pp. 533-540. 1849

[28] O. Chapelle, et al., Semi-Supervised Learning, vol. 2. Cambridge, 1850 MA, USA: MIT Press, 2006.

[29] J. Chen, X. Liu, P. Tu, and A. Aragones, "Learning person-specific 1852 models for facial expressions and action unit recognition," Pattern 1853 Recognition Lett., vol. 34, no. 15, pp. 1964-1970, 2013

[30] S. W. Chew, P. Lucey, S. Lucey, J. Saragih, J. F. Cohn, and 1855 S. Sridharan, "Person-independent facial expression detection 1856 using constrained local models," in Proc. IEEE Int. Conf. Autom. 1857 Face Gesture Recognit., 2011, pp. 915-920.

[31] S. W. Chew, P. Lucey, S. Saragih, J. F. Cohn, and S. Sridharan, “In 1859 the pursuit of effective affective computing: The relationship 1860 between features and registration," IEEE Trans. Syst., Man 1861 Cybern. Part B, vol. 42, no. 4, pp. 1006-1016, Aug. 2012.

[32] G. G. Chrysos, E. Antonakos, P. Snape, A. Asthana, and 1863 S. Zafeiriou, "A comprehensive performance evaluation of 1864 deformable face tracking "in-the-wild"," Int. J. Comput. Vis., 1865 vol. 18, pp. 1-35, 2014.

[33] W. Chu, F. De La Torre, and J. F. Cohn, "Selective transfer 1867 machine for personalized facial action unit detection," in Proc. 1868 IEEE Conf. Comput. Vis. Pattern Recognit., 2013, pp. 3515-3522. 1869

[34] W.-S. Chu, J. Zeng, F. De la Torre, J. Cohn, and D. S. Messinger, 1870 "Unsupervised synchrony discovery in human interaction," in 1871 Proc. IEEE Int. Conf. Comput. Vis., 2015, pp. 3146-3154. 1872

[35] J. F. Cohn and F. De la Torre, "Automated face analysis for affec- 1873 tive computing," in The Oxford Handbook of Affective Computing. 1874 Oxford, U.K.: Oxford Univ. Press, 2014, Art. no. 131. 
[36] J. F. Cohn and P. Ekman, "Measuring facial actions," in The New Handbook of Methods in Nonverbal Behavior Research, J. A. Harrigan, R. Rosenthal, and K. Scherer, Eds. Oxford, U.K.: Oxford Univ. Press, 2005, pp. 9-64.

[37] J. F. Cohn and M. A. Sayette, "Spontaneous facial expression in a small group can be automatically measured: An initial demonstration," Behavior Res. Methods, vol. 42, no. 4, pp. 1079-1086, 2010.

[38] J. F. Cohn and K. L. Schmidt, "The timing of facial motion in posed and spontaneous smiles," J. Wavelets, Multiresolution Inform. Process., vol. 2, no. 2, pp. 121-132, 2004.

[39] T. Cootes, M. Ionita, C. Lindner, and P. Sauer, "Robust and accurate shape model fitting using random forest regression voting," in Proc. Eur. Conf. Comput. Vis., 2012, pp. 278-291.

[40] T. Cootes and C. Taylor, "Statistical models of appearance for computer vision, “ University of Manchester, Manchester, U.K., 2004.

[41] T. F. Cootes, G. J. Edwards, and C. J. Taylor, "Active appearance models," IEEE Trans. Pattern Anal. and Mach. Intell., vol. 23, no. 6, pp. 681-685, Jun. 2001

[42] T. F. Cootes, C. J. Taylor, D. H. Cooper, and J. Graham, "Active shape models-their training and application," Comput. Vis. Image Understanding, vol. 61, no. 1, pp. 38-59, 1995.

[43] C. A. Corneanu, M. Oliu, J. F. Cohn, and S. Escalera, "Survey on RGB, 3D, thermal, and multimodal approaches for facial expression recognition: History, trends, and affect-related applications," IEEE Trans. Pattern Anal. Mach. Intell., vol. 38, no. 8, pp. 1548-1568, Aug. 2016

[44] D. Cosker, E. Krumhuber, and A. Hilton, "A FACS valid 3D dynamic action unit database with applications to 3D dynamic morphable facial modeling," in Proc. IEEE Int. Conf. Comput. Vision, 2011, pp. 2296-2303.

[45] M. Costa, W. Dinsbach, A. S. R. Manstead, and P. E. R. Bitti, "Social presence, embarrassment, and nonverbal behaviour," J. Nonverbal Behav., vol. 25, no. 4, pp. 225-240, 2001.

[46] N. Dalal and B. Triggs, "Histograms of oriented gradients for human detection," in Proc. IEEE Int. Conf. Comput. Vis. Pattern Recognit., 2005, pp. 886-893.

[47] A. Dapogny, K. Bailly, and S. Dubuisson, "Dynamic facial expression recognition by joint static and multi-time gap transition classification," in Proc. IEEE Int. Conf. Workshops Autom. Face Gesture Recognit., 2015.

[48] C. Darwin, The Expression of the Emotions in Man and Animals. London, U.K.: John Murray, 1872.

[49] F. De la Torre, J. Campoy, Z. Ambadar, and J. F. Cohn, "Temporal segmentation of facial behavior," in Proc. IEEE Int. Conf. Comput. Vis., 2007, pp. 1-8.

[50] F. De la Torre and J. F. Cohn, Facial Expression Analysis. Berlin, Germany: Springer, 2011.

[51] X. Ding, W.-S. Chu, F. D. la Torre, J. F. Cohn, and Q. Wang, "Facial action unit event detection by cascade of tasks," in Proc. IEEE Int. Conf. Comput. Vision, 2013, pp. 2400-2407.

[52] P. Dollár, P. Welinder, and P. Perona, "Cascaded pose regression," in Proc. IEEE Comput. Soc. Conf. Comput. Vis. Pattern Recognit., 2010, pp. 1078-1085.

[53] G. Donato, M. S. Bartlett, J. C. Hager, P. Ekman, and T. J. Sejnowski, "Classifying facial actions," IEEE Trans. Pattern Anal. Mach. Intell., vol. 21, no. 10, pp. 974-989, Oct. 1999.

[54] G. B. Duchenne, "Mécanisme de la physionomie humaine, ou analyse électro-physiologique de ses différents modes de l'expression," Archives générales de médecine, 1862, pp. 29-47.

[55] J. Egede, M. Valstar, and B. Martinez, "Fusing deep learned and hand-crafted features of appearance, shape, and dynamics for automatic pain estimation," arXiv:1701.04540, 2017.

[56] P. Ekman, "Darwin, deception, and facial expression," Ann. New York Academy Sci., vol. 1000, pp. 205-221, 2003.

[57] P. Ekman, W. Friesen, and J. C. Hager, Facial Action Coding System. A Human Face, 2002.

[58] P. Ekman and W. V. Friesen, Facial Action Coding System: A Technique for the Measurement of Facial Movement. Washington, DC, USA: Consulting Psychologists Press, 1978.

[59] P. Ekman and L. E. Rosenberg, What the Face Reveals: Basic and Applied Studies of Spontaneous Expression Using the Facial Action Coding System. Oxford, U.K.: Oxford Univ. Press, 2005.

[60] B. Fasel and J. Luettin, "Recognition of asymmetric facial action unit activities and intensities," in Proc. Int. Conf. Pattern Recognit., 2000, pp. 1100-1103

[61] B. Fasel and J. Luettin, "Automatic facial expression analysis: A survey," Pattern Recognit., vol. 36, no. 1, pp. 259-275, 2003.
[62] P. F. Felzenszwalb, R. B. Girshick, D. A. McAllester, and 1954 D. Ramanan, "Object detection with discriminatively trained 1955 part-based models," IEEE Trans. Pattern Anal. Mach. Intell., 1956 vol. 32, no. 9, pp. 1627-1645, Sep. 2010.

[63] M. G. Frank and P. Ekman, "The ability to detect deceit general- 1958 izes across different types of high-stakes lies," J. Personality Social 1959 Psychology, vol. 72, no. 6, pp. 1429-1439, 1997.

[64] M. G. Frank and P. Ekman, "Appearing truthful generalizes 1961 across different deception situations," J. Personality Social Psychol- 1962 ogy, vol. 86, pp. 486-495, 2004.

[65] M. G. Frank, P. Ekman, and W. V. Friesen, “Behavioral markers 1964 and recognizability of the smile of enjoyment," J. Personality 1965 Social Psychology, vol. 64, no. 1, pp. 83-93, 1993.

[66] T. Gehrig and H. K. Ekenel, "Facial action unit detection using 1967 kernel partial least squares," in Proc. IEEE Int. Conf. Comput. Vis. 1968 Workshop, 2011, pp. 2092-2099.

[67] G. Ghiasi and C. C. Fowlkes, "Occlusion coherence: Localizing 1970 occluded faces with a hierarchical deformable part model," in 1971 Proc. IEEE Int. Conf. Comput. Vis. Pattern Recognit., 2014, 1972 pp. 1899-1906.

[68] J. M. Girard, W.-S. Chu, L. A. Jeni, and J. F. Cohn, "Sayette group 1974 formation task (GFT) spontaneous facial expression database," 1975 in Proc. 12th IEEE Int. Conf. Autom. Face Gesture Recognit., 2017, 1976 pp. 581-588.

[69] J. M. Girard, J. F. Cohn, and F. D. la Torre, "Estimating smile 1978 intensity: A better way," Pattern Recognit. Lett., vol. 66, pp. 13-21, 1979 2015.

[70] I. Gonzalez, H. Sahli, V. Enescu, and W. Verhelst, “Context-inde- 1981 pendent facial action unit recognition using shape and Gabor 1982 phase information," in Proc. Int. Conf. Affective Comput. Intell. 1983 Interaction, 2011, pp. 548-557.

[71] A. Gudi, H. E. Tasli, T. M. den Uyl, and A. Maroulis, “Deep 1985 learning based facs action unit occurrence and intensity 1986 estimation," in Proc. IEEE Int. Conf. Autom. Face Gesture Recognit. 1987 Workshop, 2015, pp. 1-5.

[72] N. Haber, C. Voss, A. Fazel, T. Winograd, and D. P. Wall, 1989 "A practical approach to real-time neutral feature subtraction for 1990 facial expression recognition," in Proc. IEEE Winter Conf. Appl. 1991 Comput. Vis., 2016, pp. 1-9.

[73] J. Hamm, C. G. Kohler, R. C. Gur, and R. Verma, "Automated 1993 facial action coding system for dynamic analysis of facial expres- 1994 sions in neuropsychiatric disorders," J. Neuroscience Methods, 1995 vol. 200, no. 2, pp. 237-56, 2011

[74] T. Hassner, S. Harel, E. Paz, and R. Enbar, "Effective face frontal- 1997 ization in unconstrained images," in Proc. IEEE Int. Conf. Comput. 1998 Vis. Pattern Recognit., 2015, pp. 4295-4304.

[75] J. He, L. Dongliang C. Siming S. Bo, and Y. Lejun, "Multi view 2000 facial action unit detection based on CNN and BLSTM-RNN," in 2001 Proc. IEEE Int. Conf. Autom. Face Gesture Recognit., 2017, pp. 848-853. 2002

[76] M. Heller and V. Haynal, "The faces of suicidal depression," Kah- 2003 iers Psychiatriques Genevois, vol. 16, pp. 107-117, 1994.

[77] G. E. Hinton, "Training products of experts by minimizing con- 2005 trastive divergence," Neural Comput., vol. 14, no. 8, pp. 1771- 2006 1800, 2002.

[78] S. Honari, J. Yosinski, P. Vincent, and C. Pal, "Recombinator net- 2008 works: Learning coarse-to-fine feature aggregation," in IEEE Int. 2009 Conf. Comput. Vis. Pattern Recognit., 2016, pp. 5743-5752.

D. Huang, C. Shan, and M. Ardabilian, "Localbina its application to facial image analysis: A survey" IEEE Trans. 2012 Syst., Man Cybernet. Part C, vol. 41, pp. 765-781, Nov. 2011.

[80] S. Jaiswal, B. Martinez, and M. F. Valstar "Learning to combine 2014 local models for facial action unit detection," in IEEE Int. Conf. 2015 Autom. Face Gesture Recognit. Workshop, 2015, pp. 1-6.

[81] S. Jaiswal and M. F. Valstar, “Deep learning the dynamic appear- 2017 ance and shape of facial action units," in Proc. IEEE Winter Conf. 2018 Appl. Comput. Vis., 2016, pp. 1-8.

[82] S. Jarlier, et al., "Thermal analysis of facial muscles contractions," 2020 IEEE Trans. Affective Comput., vol. 2, no. 1, pp. 2-9, Jan.-Mar. 2011. 2021

[83] L. Jeni, A. Lrincz, Z. Szabó, J. F. Cohn, and T. Kanade, "Spatio- 2022 temporal event classification using time-series kernel based 2023 structured sparsity," in Proc. Eur. Conf. Comput. Vis., 2014, 2024 pp. $135-150$.

[84] L. A. Jeni, J. F. Cohn, and F. De la Torre, "Facing imbalanced 2026 data-recommendations for the use of performance metrics," in 2027 Proc. Conf. Affective Comput. Intell. Interaction, 2013, pp. 245-251. 2028

[85] L. A. Jeni, J. F. Cohn, and T. Kanade, "Dense 3d face alignment 2029 from $2 \mathrm{~d}$ video for real-time use," Image Vis. Comput., vol. 58, 2030 pp. 13-24, 2017. 
[86] L. A. Jeni, J. M. Girard, J. Cohn, and F. D. L. Torres, “Continuous AU intensity estimation using localized, sparse facial feature space," in Proc. IEEE Int. Conf. Workshops Autom. Face Gesture Recognit., 2013, pp. 1-7.

[87] B. Jiang, B. Martinez, and M. Pantic, "Parametric temporal alignment for the detection of facial action temporal segments," in Proc. Brit. Mach. Vis. Conf., 2014, pp. 1-11.

[88] B. Jiang, M. F. Valstar, B. Martinez, and M. Pantic, "Dynamic appearance descriptor approach to facial actions temporal modelling," IEEE Trans. Syst., Man Cybern., Part B, vol. 44, no. 2, pp. 161-174, Feb. 2014

[89] S. E. Kahou, X. Bouthillier, P. Lamblin, and C. E. A. Gulcehre, "Emonets: Multimodal deep learning approaches for emotion recognition in video," J. Multimodal User Interfaces, vol. 10, no. 2, pp. 99-111, 2016.

[90] S. Kaltwang, O. Rudovic, and M. Pantic, "Continuous pain intensity estimation from facial expressions," in Proc. Int. Symp. Visual Comput., 2012, pp. 368-377.

[91] S. Kaltwang, S. Todorovic, and M. Pantic, "Latent trees for estimating intensity of facial action units," in Proc. IEEE Conf. Comput. Vis. Pattern Recognit., 2015, pp. 296-304.

[92] T. Kanade, J. F. Cohn, and Y. Tian, "Comprehensive database for facial expression analysis," in Proc. IEEE Int. Conf. Autom. Face Gesture Recognit., 2000, pp. 46-53.

[93] V. Kazemi and J. Sullivan, "One millisecond face alignment with an ensemble of regression trees," in Proc. IEEE Conf. Comput. Vis. Pattern Recognit., 2014, pp. 1867-1874.

[94] S. Koelstra, M. Pantic, and I. Patras, "A dynamic texture based approach to recognition of facial actions and their temporal models," IEEE. Trans. Pattern Anal. Mach. Intell., vol. 32, no. 11, pp. 1940-1954, Nov. 2010.

[95] I. Kotsia, S. Zafeiriou, and I. Pitas, "Texture and shape information fusion for facial expression and facial action unit recognition," Pattern Recognit., vol. 41, no. 3, pp. 833-851, 2008

[96] F. D. la Torre and J. F. Cohn, "Facial expression analysis," in Visual Analysis of Humans. Berlin, Germany: Springer, 2011, pp. 377-409.

[97] H. Li, Z. Lin, X. Shen, J. Brandt, and G. Hua, "A convolutional neural network cascade for face detection," in Proc. IEEE Conf. Comput. Vis. Pattern Recognit., 2015, pp. 5325-5334.

[98] X. Li, S. Chen, and Q. Jin, "Facial action units detection with multi-features and -AUs fusion," in Proc. IEEE Int. Conf. Autom. Face Gesture Recognit., 2017, pp. 860-865.

[99] Y. Li, S. M. Mavadati, M. H. Mahoor, Y. Zhao, and Q. Ji, "Measuring the intensity of spontaneous facial action units with dynamic Bayesian network," Pattern Recognit., vol. 48, no. 11, pp. 3417-3427, 2015.

[100] J. J. Lien, T. Kanade, J. F. Cohn, and C. Li, "Automated facial expression recognition based on FACS action units," in Proc. IEEE Int. Conf. Autom. Face Gesture Recognit., 1998, pp. 390-395.

[101] J. J. Lien, T. Kanade, J. F. Cohn, and C. Li, "Detection, tracking, and classification of action units in facial expression," Robot. Autonom. Sys., vol. 31, pp. 131-146, 2000.

[102] J.-C. Lin, C.-H. Wu, and W.-L. Wei, "Facial action unit prediction under partial occlusion based on error weighted cross-correlation model," in Proc. Int. Conf. Acoust., Speech Signal Process., 2013, pp. 3482-3486.

[103] P. Liu, S. Han, Z. Meng, and Y. Tong, "Facial expression recognition via a boosted deep belief network," in Proc. IEEE Conf. Comput. Vis. Pattern Recognit., 2014, pp. 1805-1812.

[104] D. G. Lowe, "Distinctive image features from scale-invariant keypoints," Int. J. Comput. Vision, vol. 60, no. 2, pp. 91-110, 2004.

[105] P. Lucey, J. F. Cohn, T. Kanade, J. Saragih, and Z. Ambadar, "The extended cohn-kanade dataset $(\mathrm{CK}+)$ : A complete dataset for action unit and emotion-specified expression," in Proc. IEEE Comput. Soc. Conf. Comput. Vis. Pattern Recognit.- Workshop, 2010, pp. 94-101.

[106] P. Lucey, et al., "Automatically detecting pain in video through facial action units," IEEE Trans. Syst., Man Cybern. Part B, vol. 41, pp. 664-674, Jun. 2011

[107] P. Lucey, J. F. Cohn, K. M. Prkachin, P. E. Solomon, and I. Matthews, "Painful data: The UNBC-McMaster shoulder pain expression archive database," in Proc. IEEE Int. Conf. Autom. Face Gesture Recognit., 2011, pp. 57-64.

[108] M. H. Mahoor, S. Cadavid, D. S. Messinger, and J. F. Cohn, "A framework for automated measurement of the intensity of non-posed facial action units," in Proc. IEEE Comput. Vis. Pattern Recognit.- Workshop, 2009, pp. 74-80.
[109] M. H. Mahoor, M. Zhou, K. L. Veon, M. Mavadati, and J. F. Cohn, 2110 "Facial action unit recognition with sparse representation," in Proc. 2111 IEEE Int. Conf. Autom. Face Gesture Recognit., 2011, pp. 336-342.

[110] B. Martinez and M. F. Valstar, "Advances, challenges, and 2113 opportunities in automatic facial expression recognition," in 2114 Advances in Face Detection and Facial Image Analysis. Berlin, 2115 Germany: Springer, 2016, pp. 63-100.

[111] B. Martinez, M. F. Valstar, X. Binefa, and M. Pantic, "Local evi- 2117 dence aggregation for regression based facial point detection," 2118 IEEE Trans. Pattern Anal. Mach. Intell., vol. 35, no. 5, pp. 1149- 2119 1163, May 2013.

[112] M. Mathias, R. Benenson, M. Pedersoli, and L. Van Gool, "Face 2121 detection without bells and whistles," in Proc. Eur. Conf. Comput. 2122 Vis., 2014, pp. 720-735.

[113] I. Matthews and S. Baker, "Active appearance models revisited," 2124 Int. J. Comput. Vis., vol. 60, no. 2, pp. 135-164, 2004.

[114] S. Mavadati and M. Mahoor, "Temporal facial expression model- 2126 ing for automated action unit intensity measurement," in Proc. 2127 Int. Conf. Pattern Recognit., 2014, pp. 4648-4653.

[115] S. M. Mavadati, M. H. Mahoor, K. Bartlett, and P. Trinh, 2129 "Automatic detection of non-posed facial action units," in Proc. 2130 Int. Conf. Image Process., 2012, pp. 1817-1820.

[116] A. McCallum, D. Freitag, and F. C. N. Pereira, "Maximum entropy 2132 Markov models for information extraction and segmentation," in 2133 Proc. Int. Conf. Mach. Learning, 2000, pp. 591-598.

2134

[117] D. McDuff, R. Kaliouby, T. Senechal, M. Amr, J. F. Cohn, and 2135 R. Picard, "Affectiva-MIT facial expression dataset (AM-FED): 2136 Naturalistic and spontaneous facial expressions collected in-the- 2137 wild," in Proc. IEEE Conf. Comput. Vis. Pattern Recognit.- Work- 2138 shop, 2013, pp. 881-888.

[118] G. McKeown, M. F. Valstar, R. Cowie, M. Pantic, and 2140 M. Schroder, "The SEMAINE database: Annotated multimodal 2141 records of emotionally colored conversations between a person 2142 and a limited agent," IEEE Trans. Affective Comput., vol. 3, no. 1, 2143 pp. 5-17, Jan.-Mar. 2012.

[119] T. McLellan, L. Johnston, J. Dalrymple-Alford, and R. Porter, 2145 "Sensitivity to genuine versus posed emotion specified in facial 2146 displays," Cognition Emotion, vol. 24, pp. 1277-1292, 2010.

[120] S. Milborrow and F. Nicolls, "Locating facial features with an 2148 extended active shape model," in Proc. Eur. Conf. Comput. Vision, 2149 2008, pp. 504-513.

2150

[121] M. Mohammadi, E. Fatemizadeh, and M. Mahoor, "Intensity 2151 estimation of spontaneous facial action units based on their spar- 2152 sity properties," IEEE Trans. Cybern., vol. 46, no. 3, pp. 817-826, 2153 Mar. 2016.

[122] H.-W. Ng, V. D. Nguyen, V. Vonikakis, and S. Winkler, “Deep 2155 learning for emotion recognition on small datasets using transfer 2156 learning," in Proc. Int. Conf. Multimodal Interfaces, 2015, pp. 443- 2157 449.

[123] M. H. Nguyen, L. Torresani, F. de la Torre, and C. Rother, 2159 "Weakly supervised discriminative localization and classifica- 2160 tion: A joint learning process," in Proc. IEEE Int. Conf. Comput. 2161 Vision, 2009, pp. 1925-1932.

[124] C. Nhan Duong, K. Luu, K. Gia Quach, and T. D. Bui, “Beyond 2163 principal components: Deep boltzmann machines for face mod- 2164 eling," in Proc. IEEE Conf. Comput. Vis. Pattern Recognit., 2015, 2165 pp. $4786-4794$

[125] T. Ojala, M. Pietikainen, and D. Harwood, "A comparative study 2167 of texture measures with classification based on featured distri- 2168 bution," Pattern Recognit., vol. 29, no. 1, pp. 51-59, 1996.

2169

[126] T. Ojala, M. Pietikainen, and T. Maenpaa, "Multiresolution grey- 2170 scale and rotation invariant texture classification with local 2171 binary patterns," IEEE Trans. Pattern Anal. Mach. Intell., vol. 24, 2172 no. 7, pp. 971-987, Jul. 2002.

2173

[127] V. Ojansivu and J. Heikkila, "Blur insensitive texture classifica- 2174 tion using local phase quantization," in Proc. Int. Conf. Image Sig- 2175 nal Process., 2008, pp. 236-243.

[128] J. Orozco, B. Martinez, and M. Pantic, "Empirical analysis of cas- 2177 cade deformable models for multi-view face detection," Image 2178 Vis. Comput., vol. 42, pp. 47-61, 2015.

[129] S. J. Pan and Q. Yang, "A survey on transfer learning," IEEE 2180 Trans. Knowl. Data Eng., vol. 22, no. 10, pp. 1345-1359, Oct. 2010.2181

[130] M. Pantic, "Machine analysis of facial behaviour: Naturalistic 2182 and dynamic behaviour," Philosoph. Trans. Roy. Soc. B: Biological 2183 Sci., vol. 365, no. 1535, pp. 3505-3513, 2009.

[131] M. Pantic and M. S. Bartlett, Machine Analysis of Facial Expressions. 2185 Vienna, Austria: I-Tech Education Publishing, 2007, ch. 20, 2186 pp. 377-416. 
[132] M. Pantic and I. Patras, "Temporal modeling of facial actions from face profile image sequences," in Proc. Int. Conf. Multimedia Expo, 2004, pp. 49-52.

[133] M. Pantic and J. Rothkrantz, "Automatic analysis or facial expressions: The state of the art," IEEE Trans. Pattern Anal. Mach. Intell., vol. 22, no. 12, pp. 1424-1445, Dec. 2000.

[134] M. Pantic, L. Rothkrantz, and H. Koppelaar, "Automation of non-verbal communication of facial expressions," in Proc. Eur. Conf. Multimedia, 1998, pp. 86-93.

[135] M. Pantic, M. F. Valstar, R. Rademaker, and L. Maat, "Web-based database for facial expression analysis," in Proc. Int. Conf. Multimedia Expo, 2005, pp. 317-321.

[136] C. P. Papageorgiou, M. Oren, and T. Poggio, "A general framework for object detection," in Proc. IEEE Int. Conf. Comput. Vis., 1998, pp. 555-562.

[137] X. Peng, R. S. Feris, X. Wang, and D. N. Metaxas, "A recurrent encoder-decoder network for sequential face alignment," in Proc. Eur. Conf. Comput. Vis., 2016, pp. 38-56.

[138] X. Peng, S. Zhang, Y. Yang, and D. N. Metaxas, "PIEFA: Personalized incremental and ensemble face alignment," in Proc. IEEE Int. Conf. Comput. Vis., 2015, pp. 3880-3888.

[139] T. Pfister, X. Li, G. Zhao, and M. Pietikäinen, "Recognising spontaneous facial micro-expressions," in Proc. IEEE Int. Conf. Comput. Vis., 2011, pp. 1449-1456.

[140] S. Ren, X. Cao, Y. Wei, and J. Sun, "Face alignment at $3000 \mathrm{fps}$ via regressing local binary features," in Proc. IEEE Conf. Comput. Vis. Pattern Recognit., 2014, pp. 1685-1692.

[141] F. Ringeval, E. Marchi, M. Mehu, K. Scherer, and B. Schuller, "Face reading from speech - predicting facial action units from audio cues," in Proc. INTERSPEECH, 2015, pp. 1977-1981.

[142] W. E. Rinn, "The neuropsychology of facial expression: A review of the neurological and psychological mechanisms for producing facial expressions," Psychological Bulletin, vol. 95, no. 1, 1984, Art. no. 52.

[143] B. Romera-Paredes, H. Aung, N. Bianchi-Berthouze, and M. Pontil, "Multilinear multitask learning," in Proc. IEEE Int. Conf. Mach. Learn., 2013, pp. 1444-1452.

[144] O. Rudovic, V. Pavlovic, and M. Pantic, "Kernel conditional ordinal random fields for temporal segmentation of facial action units," in Proc. Eur. Conf. Comput. Vis. Workshop, 2012, pp. 260-269.

[145] A. Ruiz, J. V. de Weijer, and X. Binefa, "Regularized multi-concept mil for weakly-supervised facial behavior categorization," in Proc. Brit. Mach. Vis. Conf., 2014, pp. 1-12.

[146] A. Ruiz, J. Van de Weijer, and X. Binefa, "From emotions to action units with hidden and semi-hidden-task learning," in Proc. IEEE Int. Conf. Comput. Vis., 2015, pp. 3703-3711.

[147] C. Sagonas, Y. Panagakis, S. Zafeiriou, and M. Pantic, "RAPS: Robust and efficient automatic construction of person-specific deformable models," in Proc. IEEE Conf. Comput. Vis. Pattern Recognit., 2014, pp. 1789-1796.

[148] C. Sagonas, Y. Panagakis, S. Zafeiriou, and M. Pantic, "Robust statistical face frontalization," in Proc. IEEE Int. Conf. Comput. Vis., 2015, pp. 3871-3879.

[149] E. Sánchez-Lozano, B. Martinez, G. Tzimiropoulos, and M. Valstar, "Cascaded continuous regression for real-time incremental face tracking," in Proc. Eur. Conf. Comput. Vis., 2016, pp. 645-661.

[150] G. Sandbach, S. Zafeiriou, and M. Pantic, "Markov random field structures for facial action unit intensity estimation," in Proc. IEEE Int. Conf. Comput. Vis.- Workshop, 2013, pp. 738-745.

[151] G. Sandbach, S. Zafeiriou, M. Pantic, and L. Yin, "Static and dynamic 3D facial expression recognition: A comprehensive survey," Image Vis. Comput., vol. 30, no. 10, pp. 683-697, 2012.

[152] E. Sangineto, G. Zen, E. Ricci, and N. Sebe, "We are not all equal: Personalizing models for facial expression analysis with transductive parameter transfer," in Proc. ACM Int. Conf. Multimedia, 2014, pp. 357-366.

[153] J. M. Saragih, S. Lucey, and J. F. Cohn, "Deformable model fitting by regularized landmark mean-shift," Int. J. Comput. Vision, vol. 91, no. 2, pp. 200-215, 2011.

[154] E. Sariyanidi, H. Gunes, and A. Cavallaro, "Automatic analysis of facial affect: A survey of registration, representation, and recognition," IEEE Trans. Pattern Anal. Mach. Intell., vol. 37, no. 6, pp. 1113-1133, Jun. 2015.

[155] A. Savran, et al., "Bosphorus database for 3D face analysis," in Proc. COST Workshop Biometrics Identity Manag., 2008, pp. 47-56.
[156] A. Savran, B. Sankur, and M. T. Bilge, "Comparative evaluation 2266 of 3D versus 2D modality for automatic detection of facial action 2267 units," Pattern Recog., vol. 45, no. 2, pp. 767-782, 2012.

[157] A Savran, B. Sankur, and M. T. Bilge "Regression-based inten- 2269 sity estimation of facial action units," Image Vis. Comput., vol. 30, 2270 no. 10 , pp. 774-784, 2012

[158] K. Scherer and P. Ekman, Handbook of Methods in Nonverbal Behav- 2272 ior Research. Cambridge, U.K.: Cambridge Univ. Press, 1982.

[159] S. Scherer, et al., "Automatic audiovisual behavior descriptors 2274 for psychological disorder analysis," Image Vis. Comput., vol. 32, 2275 pp. 648-658, 2014.

[160] T. Senechal, V. Rapp, H. Salam, R. Seguier, K. Bailly, and 2277 L. Prevost, "Combining AAM coefficients with LGBP histograms 2278 in the multi-kernel SVM framework to detect facial action units," 2279 in Proc. IEEE Int. Conf. Autom. Face Gesture Recognit. Workshop, 2280 2011, pp. 860-865.

[161] T. Senechal, V. Rapp, H. Salam, R. Seguier, K. Bailly, and 2282 L. Prevost, "Facial action recognition combining heterogeneous 2283 features via multi-kernel learning," IEEE Trans. Syst. Man 228 Cybern., Part B, vol. 42, no. 4, pp. 993-1005, Aug. 2012.

[162] C. Shan, S. Gong, and P. McOwan, "Facial expression recognition 2286 based on local binary patterns: A comprehensive study," Image 2287 Vis. Comput., vol. 27, no. 6, pp. 803-816, 2008.

[163] J. Shen, S. Zafeiriou, G. G. Chrysos, J. Kossaifi, G. Tzimiropoulos, 2289 and M. Pantic, "The first facial landmark tracking in-the-wild 2290 challenge: Benchmark and results," in Proc. IEEE Int. Conf. Com- 2291 put. Vis. Workshop, 2015, pp. 1003-1011.

[164] T. Simon, M. H. Nguyen, F. D. L. Torre, and J. Cohn, "Action unit 2293 detection with segment-based SVMs," in Proc. IEEE Conf. 2294 Comput. Vis. Pattern Recognit., 2010, pp. 2737-2744.

[165] J. Sivic, B. Russell, A. Efros, A. "Discovering objects and their location in images," in Proc. IEEE 2297 Int. Conf. Comput. Vis., 2005, pp. 370-377.

[166] Y.Song, D. McDuff, D. Vasisht, and A. Kapoor, "Exploiting spar- 2299 sity and co-occurrence structure for action unit recognition," in 2300 Proc. IEEE Int. Conf. Workshops Autom. Face and Gesture Recognit., 2301 2015, pp. 1-8.

2302

[167] G. Stratou, A. Ghosh, P. Debevec, and L.-P. Morency, "Effect of 2303 illumination on automatic expression recognition: A novel 3D 2304 relightable facial database," in Proc. IEEE Int. Conf. Autom. Face 2305 Gesture Recognit., 2011, pp. 611-618.

[168] Y. Sun, X. Wang, and X. Tang, "Deep convolutional network cas- 2307 cade for facial point detection," in Proc. IEEE Conf. Comput. Vis. 2308 Pattern Recognit., 2013, pp. 3476-3483.

[169] C. Tang, et al., "View-independent facial action unit detection," in 2310 Proc. IEEE Int. Conf. Autom. Face Gesture Recognit., 2017, pp. 878-882. 2311

[170] B. Taskar, C. Guestrin, and D. Koller, "Max-margin Markov 2312 networks," in Proc. Advances Neural Inform. Process. Syst., 2003, 2313 pp. 25-32.

[171] D. M. J. Tax, E. Hendriks, M. F. Valstar, and M. Pantic, "The 2315 detection of concept frames using clustering multi-instance 2316 learning," in Proc. Int. Conf. Pattern Recognit., 2010, pp. 2917- 2317 2920.

[172] Y. Tian, T. Kanade, and J. Cohn, "Recognizing action units for 2319 facial expression analysis," IEEE Trans. Pattern Anal. Mach. Intell., 2320 vol. 23, no. 2, pp. 97-115, Feb. 2001.

[173] Y. Tian, T. Kanade, and J. F. Cohn, "Evaluation of Gabor- 2322 wavelet-based facial action unit recognition in image sequences 2323 of increasing complexity," in Proc. IEEE Int. Conf. Autom. Face 2324 Gesture Recognit., 2002, pp. 229-234.

[174] Y. Tian, T. Kanade, and J. F. Cohn, "Facial expression analysis," 2326 in Handbook of Face Recog. Berlin, Germany: Springer, ch. 11, 2005. 2327

[175] E. Tola, V. Lepetit, and P. Fua, "DAISY: An efficient dense 2328 descriptor applied to wide baseline stereo," IEEE Trans. Pattern 2329 Anal. Mach. Intell., vol. 32, no. 5, pp. 815-830, May 2010.

[176] Y. Tong, J. Chen, and Q. Ji, "A unified probabilistic framework for 233 spontaneous facial action modeling and understanding," IEEE 2332 Trans. Pattern Anal. Mach. Intell., vol. 32, no. 2, pp. 258-273, Feb. 2010. 2333

[177] Y. Tong, W. Liao, and Q. Ji, "Facial action unit recognition by 2334 exploiting their dynamic and semantic relationships," IEEE Trans. 2335 Pattern Anal. Mach. Intell., vol. 29, no. 10, pp. 1683-1699, Oct. 2007. 2336

[178] G. Trigeorgis, P. Snape, M. A. Nicolaou, E. Antonakos, and 2337 S. Zafeiriou, "Mnemonic descent method: A recurrent process 2338 applied for end-to-end face alignment," in Proc. IEEE Conf. 2339 Comput. Vis. Pattern Recognit., 2016, pp. 4177-4187.

[179] I. Tsochantaridis, T. Joachims, T. Hofmann, and Y. Altun, "Large 2341 margin methods for structured and interdependent output varia- 2342 bles," J. Mach. Learning Res., vol. 6, pp. 1453-1484, 2005. 
[180] G. Tzimiropoulos, "Project-out cascaded regression with an application to face alignment," in Proc. IEEE Conf. Comput. Vis. Pattern Recognit., 2015, pp. 3659-3667.

[181] G. Tzimiropoulos and M. Pantic, "Optimization problems for fast AAM fitting in-the-wild," in Proc. IEEE Int. Conf. Comput. Vis., 2013, pp. 593-600.

[182] G. Tzimiropoulos and M. Pantic, "Gauss-Newton deformable part models for face alignment in-the-wild," in Proc. IEEE Conf. Comput. Vis. Pattern Recognit., 2014, pp. 1851-1858.

[183] G. Tzimiropoulos and M. Pantic, "Fast algorithms for fitting active appearance models to unconstrained images," Int. J. Comput. Vis., vol. 122, no. 1, pp. 17-33, 2017.

[184] M. Valstar, et al., "Fera 2017 - addressing head pose in the third facial expression recognition and analysis challenge," in Proc. IEEE Int. Conf. Autom. Face Gesture Recognit., 2017, pp. 839-847.

[185] M. Valstar, S. Zafeiriou, and M. Pantic, "Facial action recognition in $2 \mathrm{~d}$ and $3 \mathrm{~d}, "$ in Face Recognition in Adverse Conditions. Hershey, PA, USA: IGI Global, 2014, pp. 167-186.

[186] M. F. Valstar, et al., "Fera 2015 - second facial expression recognition and analysis challenge," in Proc. IEEE Int. Conf. Workshops Autom. Face Gesture Recognit., 2015, pp. 1-8.

[187] M. F. Valstar, H. Gunes, and M. Pantic, "How to distinguish posed from spontaneous smiles using geometric features," in Proc. Int. Conf. Multimodal Interfaces, 2007, pp. 38-45.

[188] M. F. Valstar, B. Jiang, M. Mehu, M. Pantic, and K. Scherer, "The first facial expression recognition and analysis challenge," in Proc. IEEE Int. Conf. Autom. Face Gesture Recognit. Workshop, 2011, pp. 921-926.

[189] M. F. Valstar, M. Mehu, B. Jiang, M. Pantic, and K. Scherer, "Metaanalyis of the first facial expression recognition challenge," IEEE. Trans. Syst. Man Cybern. Part B, vol. 42, no. 4, pp. $966-979$, Aug. 2012.

[190] M. F. Valstar and M. Pantic, "Induced disgust, happiness and surprise: An addition to the MMI facial expression database," in Proc. Int. Conf. Language Resources Eval., 2010, pp. 65-70.

[191] M. F. Valstar and M. Pantic, "Fully automatic recognition of the temporal phases of facial actions," IEEE Trans. Syst., Man Cybern. Part B, vol. 1, no. 99, pp. 28-43, Feb. 2012.

[192] M. F. Valstar, M. Pantic, and I. Patras, "Motion history for facial action detection from face video," in Proc. IEEE Int. Conf. Syst. Man Cybern., 2004, pp. 635-640.

[193] M. F. Valstar, I. Patras, and M. Pantic, "Facial action unit detection using probabilistic actively learned support vector machines on tracked facial point data," in Proc. IEEE Comput. Soc. Conf. Comput. Vis. Pattern Recognit.- Workshop, 2005, pp. 76-76.

[194] M. F. Valstar, S. Zafeiriou, and M. Pantic, "Facial actions as social signals," in Social Signal Processing, J. K. Burgoon, N. MagnenatThalman, M. Pantic, and A. Vinciarelli, Eds. Cambridge, U.K. Cambridge Univ. Press, 2017, pp. 123-154.

[195] L. Van der Maaten and E. Hendriks, "Action unit classification using active appearance models and conditional random field," Cognitive Process., vol. 13, pp. 507-518, 2012.

[196] P. Viola and M. Jones, "Robust real-time object detection," Int. J. Comput. Vis., vol. 57, pp. 137-154, 2004.

[197] R. Walecki, O. Rudovic, V. Pavlovic, and M. Pantic, "Variablestate latent conditional random fields for facial expression recognition and action unit detection," in Proc. IEEE Int. Conf. Autom. Face Gesture Recognit., 2015, pp. 1-8.

[198] Z. Wang, Y. Li, S. Wang, and Q. Ji, "Capturing global semantic relationships for facial action unit recognition," in Proc. IEEE Int. Conf. Comput. Vis., 2013, pp. 3304-3311.

[199] J. Whitehill and C. W. Omlin, "Haar features for FACS AU recognition," in Proc. 7th IEEE Int. Conf. Autom. Face Gesture Recognit., 2006, pp. 5-101

[200] A. C. Williams, "Facial expression of pain: An evolutionary account," Behavioral Brain Sci., vol. 25, no. 4, pp. 439-488, 2002

[201] B. Wu, S. Lyu, B.-G. Hu, and Q. Ji, "Multi-label learning with missing labels for image annotation and facial action unit recognition," Pattern Recognit., vol. 48, no. 7, pp. 2279-2289, 2015.

[202] T. Wu, N. J. Butko, P. Ruvolo, J. Whitehill, M. S. Bartlett, and J. R. Movellan, "Multi-layer architectures of facial action unit recognition," IEEE Trans. Syst., Man Cybern. Part B, vol. 42, no. 4, pp. 1027-1038, Aug. 2012.

[203] X. Xiong and F. De la Torre, "Supervised descent method and its applications to face alignment," in Proc. IEEE Conf. Comput. Vis. Pattern Recognit., 2013, pp. 532-539.
[204] X. Xiong and F. De la Torre, "Global supervised descent meth- 2421 od," in Proc. IEEE Conf. Comput. Vis. Pattern Recognit., 2015, 2422 pp. 2664-2673.

[205] J. Yan, Z. Lei, D. Yi, and S. Li, "Learn to combine multiple 2424 hypotheses for accurate face alignment," in Proc. IEEE Int. Conf. 2425 Comput. Vis.- Workshop, 2013, pp. 392-396.

[206] W. Yan, et al., "CASME II: An improved spontaneous micro- 2427 expression database and the baseline evaluation," PLoS One, 2428 vol. 9, no. 1, 2014, Art. no. e86041.

[207] W. Yan, Q. Wu, Y. Liu, S. Wang, and X. Fu, "CASME database: A 2430 dataset of spontaneous micro-expressions collected from neutral- 2431 ized faces," in Proc. IEEE Int. Conf. Autom. Face Gesture Recognit., 2432 2013, pp. 1-7.

[208] P. Yang, Q. Liu, and D. N. Metaxas, "Boosting encoded dynamic 2434 features for facial expression recognition," Pattern Recognition 2435 Lett., vol. 30, no. 2, pp. 132-139, 2009.

[209] P. Yang, Q. Liu, and D. N. Metaxas, "Dynamic soft encoded pat- 2437 terns for facial event analysis," Comput. Vis. Image Understanding, 2438 vol. 115, no. 3, pp. 456-465, 2011.

[210] L. Zafeiriou, M. A. Nicolaou, S. Zafeiriou, S. Nikitidis, and 2440 M. Pantic, "Learning slow features for behaviour analysis," in 2441 Proc. IEEE Int. Conf. Comput. Vis., 2013, pp. 2840-2847. tion in the wild: Past, present and future," Comput. Vis. Image 2444 Understanding, vol. 138, pp. 1-24, 2015.

[212] G. Zen, E. Sangineto, E. Ricci, and N. Sebe, "Unsupervised 2446 domain adaptation for personalized facial emotion recognition," 2447 in Proc. IEEE Int. Conf. Multimodal Interfaces, 2014, pp. 128-135. 2448

[213] J. Zeng, W. S. Chu, F. D. 1. Torre, J. F. Cohn, and Z. Xiong, 2449 "Confidence preserving machine for facial action unit detection," 2450 in Proc. IEEE Int. Conf. Comput. Vis., 2015, pp. 3622-3630.

2451

[214] Z. Zeng, M. Pantic, G. I. Roisman, and T. S. Huang, “A survey of 2452 affect recognition methods: Audio, visual, and spontaneous 2453 expressions," IEEE Trans. Pattern Anal. Mach. Intell., vol. 31, no. 1, 2454 pp. 39-58, Jan. 2009.

2455

[215] L. Zhang, Y. Tong, and Q. Ji, "Active image labeling and its 2456 application to facial action labeling," in Proc. Eur. Conf. Comput. 2457 Vis., 2008, pp. 706-719.

[216] W. Zhang, S. Shan, W. Gao, X. Chen, and H. Zhang, "Local gabor 2459 binary pattern histogram sequence (lgbphs): A novel non-statisti- 2460 cal model for face representation and recognition," in Proc. 10th 2461 IEEE Int. Conf. Comput. Vis., 2005, pp. 786-791.

[217] X. Zhang and M. Mahoor, "Simultaneous detection of multiple 2463 facial action units via hierarchical task structure learning," in 2464 Proc. Int. Conf. Pattern Recognit., 2014, pp. 1863-1868.

2465

[218] X. Zhang, et al., "BP4D-spontaneous: A high-resolution sponta- 2466 neous 3D dynamic facial expression database," Image Vis. Com- 2467 put., vol. 32, no. 10, pp. 692-706, 2014.

[219] X. Zhang, et al., "A high-resolution spontaneous 3D dynamic 2469 facial expression database," in Proc. IEEE Int. Conf. Autom. Face 2470 Gesture Recognit., 2013, pp. 1-6.

2471

[220] Z. Zhang, P. Luo, C. C. Loy, and X. Tang, "Facial landmark detec- 2472 tion by deep multi-task learning," in Proc. Eur. Conf. Comput. Vis., 2473 2014, pp. 94-108.

[221] Z. Zhang, M. Lyons, M. Schuster, and S. Akamatsu, 2475 "Comparison between geometry-based and Gabor wavelets- 2476 based facial expression recognition using multi-layer 2477 perceptron," in Proc. IEEE Int. Conf. Autom. Face Gesture Recognit., 2478 1998 , pp. 454-459.

[222] G. Y. Zhao and M. Pietikainen, "Dynamic texture recognition 2480 using local binary pattern with an application to facial 2481 expressions," IEEE Trans. Pattern Anal. Mach. Intell., vol. 29, no. 6, 2482 pp. 915-928, Jun. 2007.

[223] K. Zhao, W.-S. Chu, F. De la Torre, J. F. Cohn, and H. Zhang, 2484 "Joint patch and multi-label learning for facial action unit 2485 detection," in Proc. IEEE Conf. Comput. Vis. Pattern Recognit., 2486 2015, pp. 2207-2216.

[224] F. Zhou, F. De la Torre, and J. F. Cohn, “Unsupervised discovery 2488 of facial events," in Proc. IEEE Conf. Comput. Vis. Pattern Recog- 2489 nit., 2010, pp. 2574-2581.

2490

[225] Y. Zhou, J. Pi, and B. Shi, "Pose-independent facial action unit 2491 intensity regression based on multi-task deep transfer learning," 2492 in Proc. IEEE Int. Conf. Autom. Face Gesture Recognit., 2017, 2493 pp. $872-877$

[226] S. Zhu, C. Li, C. Loy, and X. Tang, "Face alignment by coarse-to- 2495 fine shape searching," in Proc. IEEE Conf. Comput. Vis. Pattern 2496 Recognit., 2015, pp. 4998-5006. 
[227] X. Zhu and D. Ramanan, "Face detection pose estimation, and landmark localization in the wild," in Proc. IEEE Conf. Comput. Vis. Pattern Recognit., 2012, pp. 2879 -2886.

[228] Y. Zhu, F. De la Torre, J. F. Cohn, and Y. Zhang, "Dynamic cascades with bidirectional bootstrapping for action unit detection in spontaneous facial behavior," IEEE Trans. Affective Comput., vol. 2, no. 2, pp. 79-91, Apr.-Jun. 2011.

[229] Y. Zhu, S. Wang, L. Yue, and Q. Ji, "Multiple-facial action unit recognition by shared feature learning and semantic relation modeling," in Proc. Int. Conf. Pattern Recognit., 2014, pp. 16631668.

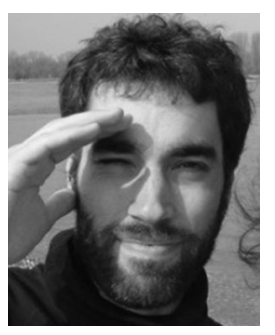

Brais Martinez (M'10) received the $\mathrm{PhD}$ degree in computer science from the Universitat Autonoma de Barcelona, and held a postdoctoral research position at the Imperial College London. $\mathrm{He}$ is a research fellow in the Computer Vision Laboratory, University of Nottingham, United Kingdom. He has worked on the topics of computer vision and pattern recognition, mainly focusing on model-free tracking and on face analysis problems such as face alignment, face detection and facial expression recognition, publishing his findings on these topics on a variety of authoritative venues such as CVPR, ICCV, ECCV or the IEEE Transactions on Pattern Analysis and Machine Intelligence. He is a member of the IEEE.

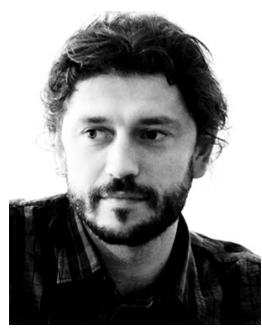

Michel F. Valstar (M'04-SM'15) received the master's degree in electrical engineering from Delft University of Technology, in 2005 and the $\mathrm{PhD}$ degree in computer science with the intelligent Behaviour Understanding Group (iBUG) from Imperial College London, in 2008. He is an associate professor in the Computer Vision and Mixed Reality Labs, University of Nottingham. His main interest is in automatic recognition of human behaviour. In 2011 he was the main organiser of the first facial expression recognition challenge, FERA 2011. In 2007 he won the BCS British Machine Intelligence Prize for part of his $\mathrm{PhD}$ work. He has published technical papers at authoritative conferences including CVPR, ICCV and SMC-B and his work has received popular press coverage in New Scientist and on BBC Radio. $\mathrm{He}$ is a senior member of the IEEE.

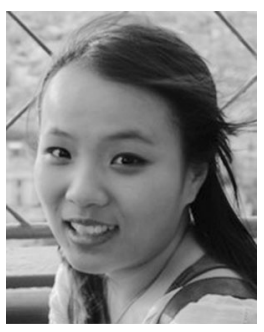

Bihan Jiang received the MSc degree in com- 2539 puter science from Imperial College London, 2540 London, United Kingdom, in 2009 and the PhD 2541 degree under the supervision of Prof. M. Pantic, 2542 in 2014. Her research interests include applying 2543 machine learning and pattern recognition 2544 approaches to the study of human (social) behav- 2545 ior and to build better human interfaces. After her 2546 $\mathrm{PhD}$ she became a financial software developer 2547 with Bloomberg LP.

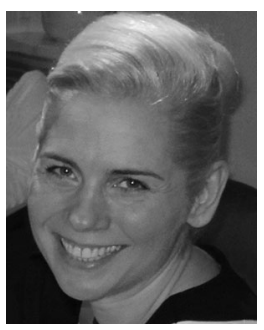

Maja Pantic (M'98-SM'06-F'12) is currently a 2549 professor in affective and behavioral computing 2550 with the Department of Computing, Imperial Col- 2551 lege London, United Kingdom, and the Depart- 2552 ment of Computer Science, University of Twente, 2553 The Netherlands. She currently serves as the 2554 editor-in-chief of the Image and Vision Computing 2555 Journal and an associate editor of the IEEE 2556 Transactions on Pattern Analysis and Machine 2557 Intelligence, and the IEEE Transactions on Affec- 2558 tive Computing. She has received various awards 2559 for her work on automatic analysis of human behavior, including the 2560 European Research Council Starting Grant Fellowship 2008 and the 2561 Roger Needham Award 2011. She is a fellow of the IEEE.

$\triangleright$ For more information on this or any other computing topic, 2563 please visit our Digital Library at www.computer.org/publications/dlib. 2564 